\title{
Ouachita Mountains Foodways: Preliminary Results from 2013-2014 Excavations at 3MN298
}

\author{
Mary Beth D. Trubitt \\ Arkansas Archeological Survey, HSU Research Station \\ Leslie L. Bush \\ Heritage Research Center, Stephen F. Austin State University \\ Lucretia S. Kelly \\ Katie Leslie
}

Follow this and additional works at: https://scholarworks.sfasu.edu/ita

Part of the American Material Culture Commons, Archaeological Anthropology Commons, Environmental Studies Commons, Other American Studies Commons, Other Arts and Humanities Commons, Other History of Art, Architecture, and Archaeology Commons, and the United States History Commons

Tell us how this article helped you.

This Article is brought to you for free and open access by the Center for Regional Heritage Research at SFA ScholarWorks. It has been accepted for inclusion in Index of Texas Archaeology: Open Access Gray Literature from the Lone Star State by an authorized editor of SFA ScholarWorks. For more information, please contact cdsscholarworks@sfasu.edu. 
Ouachita Mountains Foodways: Preliminary Results from 2013-2014 Excavations at 3MN298

\section{Creative Commons License}

(c) (1) (8)

This work is licensed under a Creative Commons Attribution-NonCommercial 4.0 International License 


\section{OUACHIta Mountains Foodways: Preliminary Results FROM 2013-2014 EXCAVATIONS AT 3MN298}

\section{Mary Beth Trubitt, Leslie L. Bush, Lucretia S. Kelly, and Katie Leslie}

New excavations in the Ouachita National Forest in west-central Arkansas, co-directed by Meeks Etchieson and Mary Beth Trubitt, are resulting in significant information about foodways of ancestral Caddo Indians living in the Ouachita Mountains region. This work has focused on the Dragover site (3MN298), located on a floodplain of the upper Ouachita River (Figures 1 and 2). Artifacts from this extensive archeological site indicate use from about 6000 B.C. to the A.D. 1900s, but it was its potential for wellpreserved organic material - animal bone, mussel shell, and charred plant seeds - that drew our research attention. Initial site testing in the 1980s uncovered several pit features with pottery sherds, chipped stone, animal bone, and mussel shells (Bennett et al. 1986). Later analysis of ceramics from those features indicated a Buckville phase Caddo occupation estimated to fall in the sixteenth century A.D. (Perttula 2009). Planting pine trees caused some damage to the site; one motivation for this project was the need for a current site evaluation so the Ouachita National Forest could better manage this historical location.

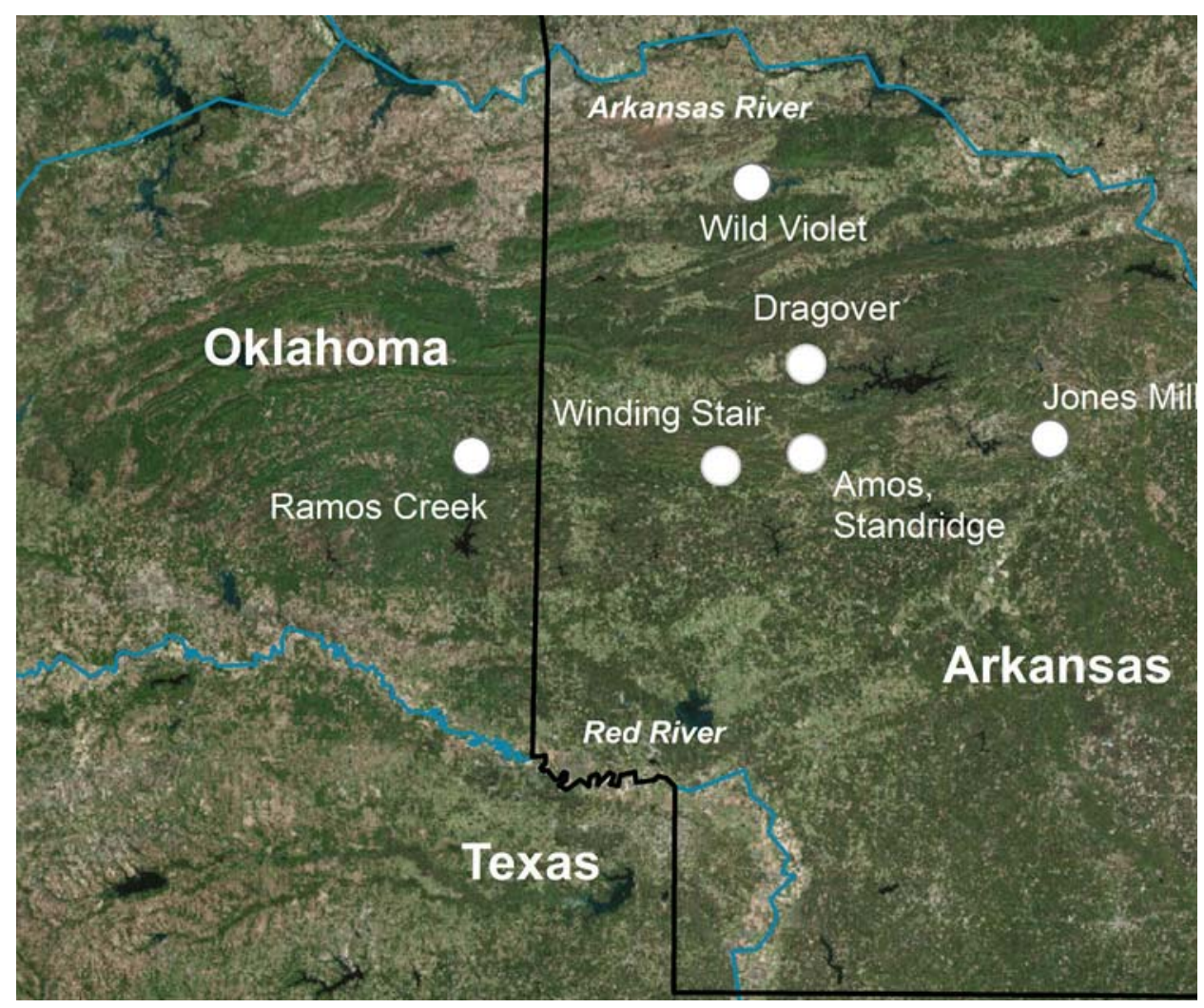

Figure 1. Location of Dragover and other archeological sites in the Ouachita Mountains region mentioned in text (map by Katie Leslie; base map uses World Imagery map layer from Environmental Systems Research Institute, Inc., and ESRI ArcGIS ArcMap 10.2.2). 


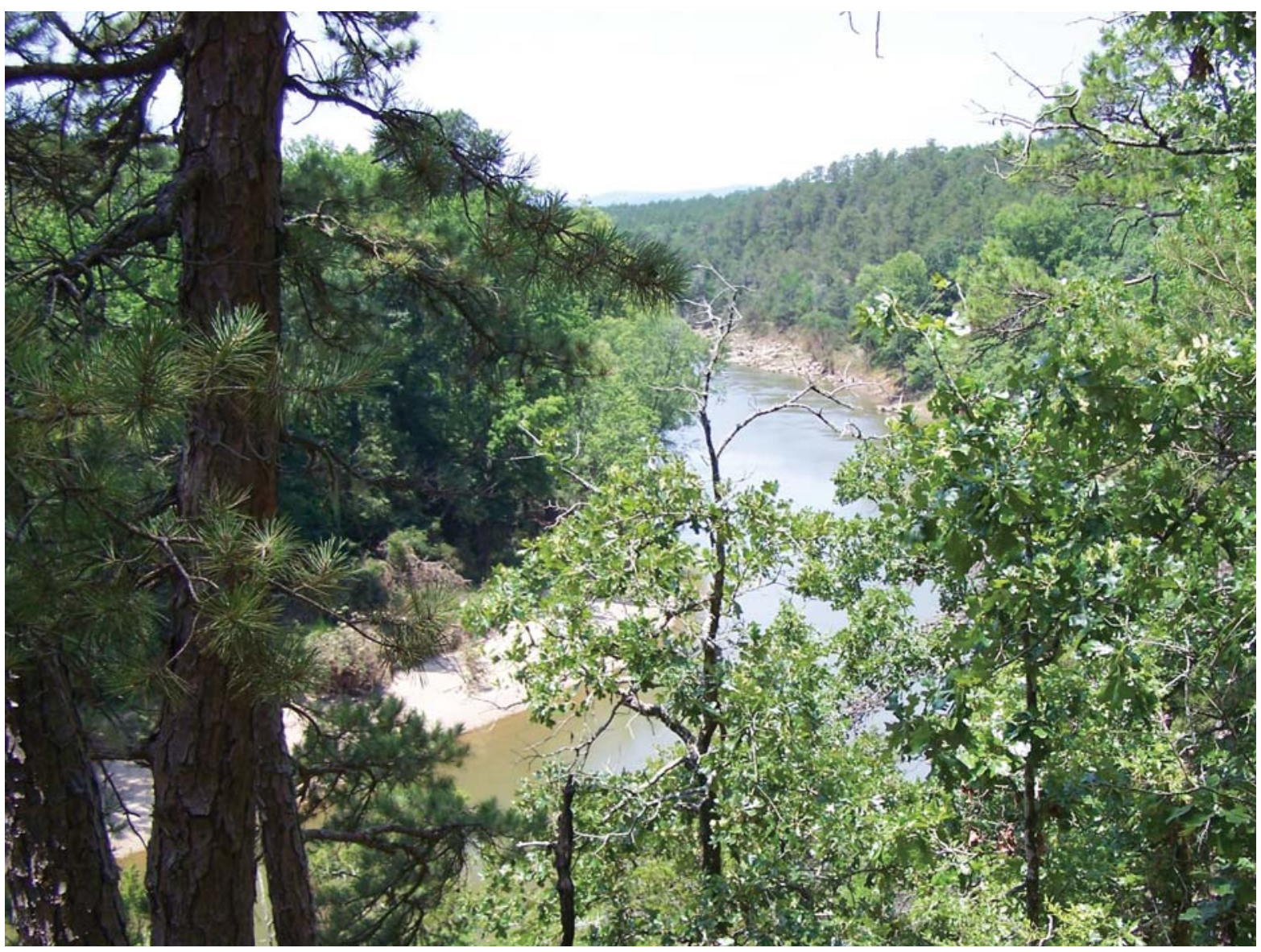

Figure 2. View of Ouachita River in Montgomery County, Arkansas (photograph by Mary Beth Trubitt). The Central Hills, Ridges, and Valleys ecoregion of the Ouachita Mountains, formed by folded and faulted layers of sandstone, shale, chert, and novaculite, is characterized by oak-hickory-pine forest (Foti and Witsell 2013; Woods et al. 2004).

Etchieson and Trubitt (2013; Trubitt and Etchieson 2014) set out a research plan that included questions about tool stone choice, plant domestication, social identity, and culture change during the expected Archaic, Woodland, and Caddo period occupations. Excavations were conducted as a cooperative endeavor between the Arkansas Archeological Survey, the U.S.D.A. Forest Service, and the Arkansas Archeological Society, with fieldwork accomplished as part of the 2013 and 2014 Society Training Programs. Key to this research has been recovering and analyzing pottery and stone tools, architectural evidence, and food remains to interpret patterns or habits of daily life in the ancient communities. Our larger research goal is to understand relationships between Caddo Indian communities in the Ouachita Mountains and communities further away in the Arkansas and Red river valleys.

Since finishing the fieldwork, we have processed soil and flotation samples and begun analyzing artifacts, and we have received results from radiocarbon dating and specialized analyses funded by the U.S.D.A. Forest Service and by three Archeological Research Fund grants from the Arkansas Archeological Society. Preliminary results have been presented at conferences and published in several short articles (Hanvey 2014; Trubitt et al. 2014), and a summary of the dating results is anticipated (Trubitt and Leslie 2016). A children's book based on the 2013 excavations, funded by an Arkansas Heritage Month grant, was produced and distributed to area schools (Ouachita Chapter 2014), and a short description of the project appears in the "Current Research" section of the Arkansas Archeological Survey's website (Trubitt and Leslie 2015). Here, we present a summary of new findings on Caddo and pre-Caddo foodways based on Dr. Leslie Bush's archeobotanical analysis and Dr. Lucretia Kelly's zooarcheological analysis from selected cultural features at this important site. 


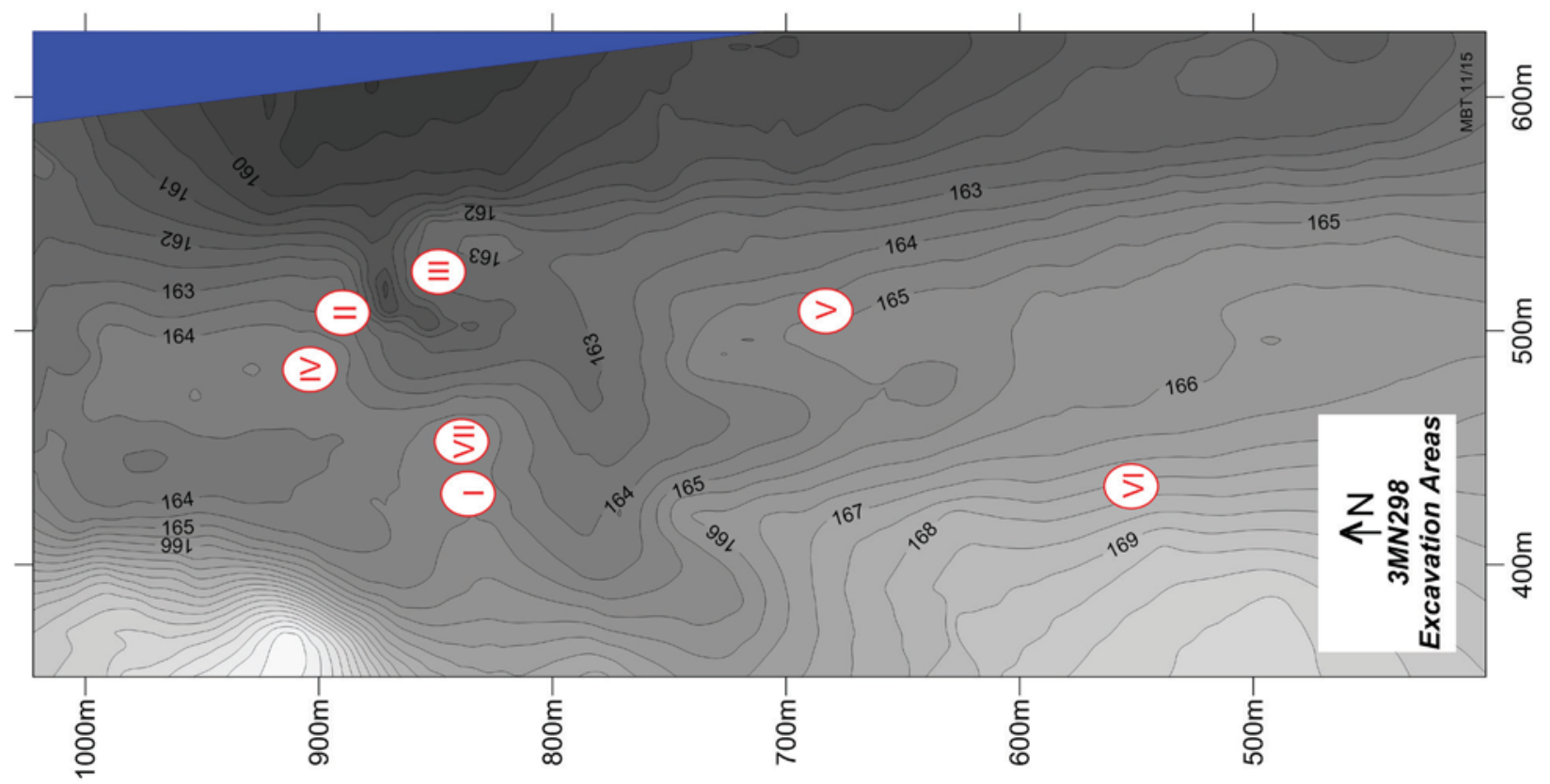

Figure 3. In 2013 and 2014, a total of $219 \mathrm{~m} 2$ was excavated in seven areas of the site. chert, and novaculite, is characterized by oak-hickory-pine forest (Foti and Witsell 2013; Woods et al. 2004).

Our excavations revealed cultural features such as clusters of fire-cracked rock (FCR) from dismantled cooking facilities, trash-filled pits, fire pits or hearths, and lines of postholes indicating house walls in six of the seven excavation areas (Figure 3). Soil samples from excavated features were processed using water flotation, and charred plant fragments identified by Leslie Bush were selected for radiocarbon dating. Eleven samples from features have been analyzed by Beta Analytic, Inc., using the accelerator mass spectrometry (AMS) technique, providing temporal detail on four main components or site occupations (Table 1 and Figure 4) (Trubitt and Leslie 2016). Late Archaic period use of the site is indicated by two dates from FCR clusters excavated in Area IV. A posthole, also in Area IV, yielded a Middle Woodland period date. A hearth or fire pit with burned clay and ash (F-82), now dated to the Late Woodland period, was uncovered in Area V (Figure 5). Six AMS dates establish a Late Caddo period component at the site. One of the postholes (F-71) associated with Structure 1 in Area V gave a date with a median probability of A.D. 1416. A large pit (F-97) in Area VII (Figure 6), filled with refuse including shell-tempered ceramic sherds and well-preserved animal bone and mussel shell, had an AMS date with a median probability of A.D. 1503. A portion of this feature had been dug and backfilled during the 1985 testing (as their Feature 3; Bennett et al. 1986). A line of postholes and a nearby hearth/burned clay concentration in Area I was designated Structure 2 (Figure 7); a combined sample of two AMS dates from features associated with Structure 2 has a median probability of A.D. 1560. A large trash-filled pit (F-11) excavated in Area I returned a date with a median probability of A.D. 1663 (but calibrated date ranges extending into the twentieth century). Further analysis will clarify the features assigned to each of these components and refine temporal interpretations. Here, we summarize the botanical analyses from the early (Archaic and Woodland periods) and late (Caddo period) components, and the analysis of animal bone from one Caddo period pit feature. 


\begin{tabular}{|c|c|c|c|c|}
\hline Provenience/Context & $\begin{array}{l}\text { Carbonized Material, } \\
\text { Isotope Ratio (d13C) }\end{array}$ & $\begin{array}{l}\text { Conventional } \\
\text { Age }\end{array}$ & $\begin{array}{l}\text { Calibrated Date, } 2 \text { sigma } \\
(95.4 \%) \text { Probability }\end{array}$ & $\begin{array}{l}\text { Median } \\
\text { Probability }\end{array}$ \\
\hline \multicolumn{5}{|l|}{ Late Archaic period: } \\
\hline $\begin{array}{l}\text { Area IV, F-2 FCR cluster, } \\
2013-325-31, \text { Beta } 402612 \\
\end{array}$ & hickory nutshell, $-25.3 \mathrm{o} / \mathrm{oo}$ & $3800+/-30 \mathrm{BP}$ & cal BC 2339-2139 (95.4\%) & cal BC 2238 \\
\hline $\begin{array}{l}\text { Area IV, F-27 FCR cluster, } \\
2013-325-33 \text {, Beta } 386085\end{array}$ & hickory nutshell, $-25.8 \mathrm{o} / \mathrm{oo}$ & $3720+/-30 \mathrm{BP}$ & cal BC 2201-2031 (95.4\%) & cal BC 2106 \\
\hline $\begin{array}{l}\text { Late Archaic Combined } \\
(\mathrm{n}=2)\end{array}$ & & $3760+/-22 \mathrm{BP}$ & $\begin{array}{l}\text { cal BC } 2280-2249(9.5 \%), \\
2231-2219(2.0 \%), 2213- \\
2131(78.0 \%), 2085-2057 \\
(6.0 \%)[\mathrm{T}=3.6, .10>\mathrm{p}>.05 \\
\mathrm{df}=1]\end{array}$ & cal BC 2172 \\
\hline \multicolumn{5}{|l|}{ Middle Woodland period: } \\
\hline $\begin{array}{l}\text { Area IV, F-34 posthole, } \\
2013-325-56, \text { Beta } 402613\end{array}$ & hickory nutshell, $-25.1 \mathrm{o} / \mathrm{oo}$ & $2140+/-30 \mathrm{BP}$ & $\begin{array}{l}\text { cal BC } 353-295(19.5 \%), \\
230-220(1.5 \%), 213-88 \\
(71.2 \%), 77-57(3.2 \%)\end{array}$ & cal BC 179 \\
\hline \multicolumn{5}{|l|}{ Late Woodland period: } \\
\hline $\begin{array}{l}\text { Area V, F-82 hearth, } \\
\text { 2013-326-120, Beta } 404379\end{array}$ & $\begin{array}{l}\text { pine bark, } \\
-26.9 \mathrm{o} / \mathrm{oo}\end{array}$ & $1280+/-30 \mathrm{BP}$ & cal AD $662-774(95.4 \%)$ & cal AD 720 \\
\hline $\begin{array}{l}\text { Area V, F-82 hearth, } \\
2013-326-122 \text {, Beta } 386086\end{array}$ & $\begin{array}{l}\text { squash flower scar, }-25.5 \\
\text { o/oo }\end{array}$ & $1200+/-30 \mathrm{BP}$ & $\begin{array}{l}\text { cal AD 715-744 (6.2\%), 765- } \\
895(87.8 \%), 928-940 \\
(1.4 \%)\end{array}$ & cal AD 825 \\
\hline Feature 82 Combined $(n=2)$ & & $1240+/-22 \mathrm{BP}$ & $\begin{array}{l}\text { cal AD } 687-779(69.8 \%), \\
790-870(25.6 \%)[\mathrm{T}=3.6, .10 \\
>\mathrm{p}>.05, \mathrm{df}=1]\end{array}$ & cal AD 743 \\
\hline \multicolumn{5}{|l|}{ Late Caddo period: } \\
\hline $\begin{array}{l}\text { Area V, F-71 posthole (Str. } \\
\text { 1), 2013-326-100, Beta } \\
404378\end{array}$ & $\begin{array}{l}\text { squash rind, } \\
-25.6 \mathrm{o} / \mathrm{oo}\end{array}$ & $520+/-30 \mathrm{BP}$ & $\begin{array}{l}\text { cal AD } 1324-1345(10.5 \%), \\
1393-1443(84.9 \%)\end{array}$ & cal AD 1416 \\
\hline $\begin{array}{l}\text { Area V, F-38 pit, } \\
\text { 2013-326-65, Beta } 364859\end{array}$ & \begin{tabular}{|l} 
corn cupules, \\
$-9.6 \mathrm{o} / \mathrm{oo}$
\end{tabular} & $420+/-30 \mathrm{BP}$ & $\begin{array}{l}\text { cal AD } 1427-1515(87.9 \%) \\
1598-1618(7.5 \%)\end{array}$ & cal AD 1460 \\
\hline $\begin{array}{l}\text { Area VII, F-97 pit (zone 4), } \\
\text { 2014-338-75, Beta } 405442\end{array}$ & $\begin{array}{l}\text { corn cupules, } \\
-9.7 \mathrm{o} / \mathrm{oo}\end{array}$ & $380+/-30 \mathrm{BP}$ & $\begin{array}{l}\text { cal AD } 1445-1524(61.6 \%), \\
1558-1632(33.8 \%)\end{array}$ & cal AD 1503 \\
\hline $\begin{array}{l}\text { Area I, F-167 hearth (Str. 2), } \\
\text { 2014-336-62, Beta } 422032\end{array}$ & $\begin{array}{l}\text { corn kernel, } \\
-9.8 \mathrm{o} / 00\end{array}$ & $370+/-30 \mathrm{BP}$ & $\begin{array}{l}\text { cal AD } 1447-1528(55.0 \%) \\
1553-1634(40.4 \%)\end{array}$ & cal AD 1515 \\
\hline $\begin{array}{l}\text { Area I, F-122 posthole (Str. } \\
\text { 2), 2014-336-19, Beta } \\
422031\end{array}$ & $\begin{array}{l}\text { black walnut shell, }-27.0 \\
\text { o/oo }\end{array}$ & $330+/-30 \mathrm{BP}$ & cal AD $1477-1643(95.4 \%)$ & cal AD 1562 \\
\hline Structure 2 Combined $(n=2)$ & & $350+/-22 \mathrm{BP}$ & $\begin{array}{l}\text { cal AD } 1461-1529(42.0 \%), \\
1543-1635(53.4 \%)[\mathrm{T}=0.9, \\
.50>\mathrm{p}>.20, \mathrm{df}=1]\end{array}$ & cal AD 1560 \\
\hline $\begin{array}{l}\text { Area I, F-11 pit, } \\
2013-322-32 \text {, Beta } 364858\end{array}$ & \begin{tabular}{||l} 
corn cupules, \\
$-9.9 \mathrm{o} / \mathrm{oo}$
\end{tabular} & $240+/-30 \mathrm{BP}$ & $\begin{array}{l}\text { calAD } 1526-1557(5.7 \%), \\
1632-1682(51.9 \%), 1738- \\
1751(1.3 \%), 1762-1803 \\
(29.5 \% .1937-(7.1 \%)\end{array}$ & cal AD 1663 \\
\hline
\end{tabular}

Table 1. Results of 3MN298 AMS dates. Conventional radiocarbon ages were reported by Beta Analytic, Inc., based on measured radiocarbon ages corrected for isotopic fractionation using delta 13C, and calibrated using the 0xCal 4.2 program (Bronk Ramsey 2009, 2015; Reimer et al. 2013). Several groups of dates were combined or averaged using 0xCal after testing for statistical difference (Drennan 1996:Table 13.4; Ward and Wilson 1978; see also Samuelsen 2014; Selden and Perttula 2013). 


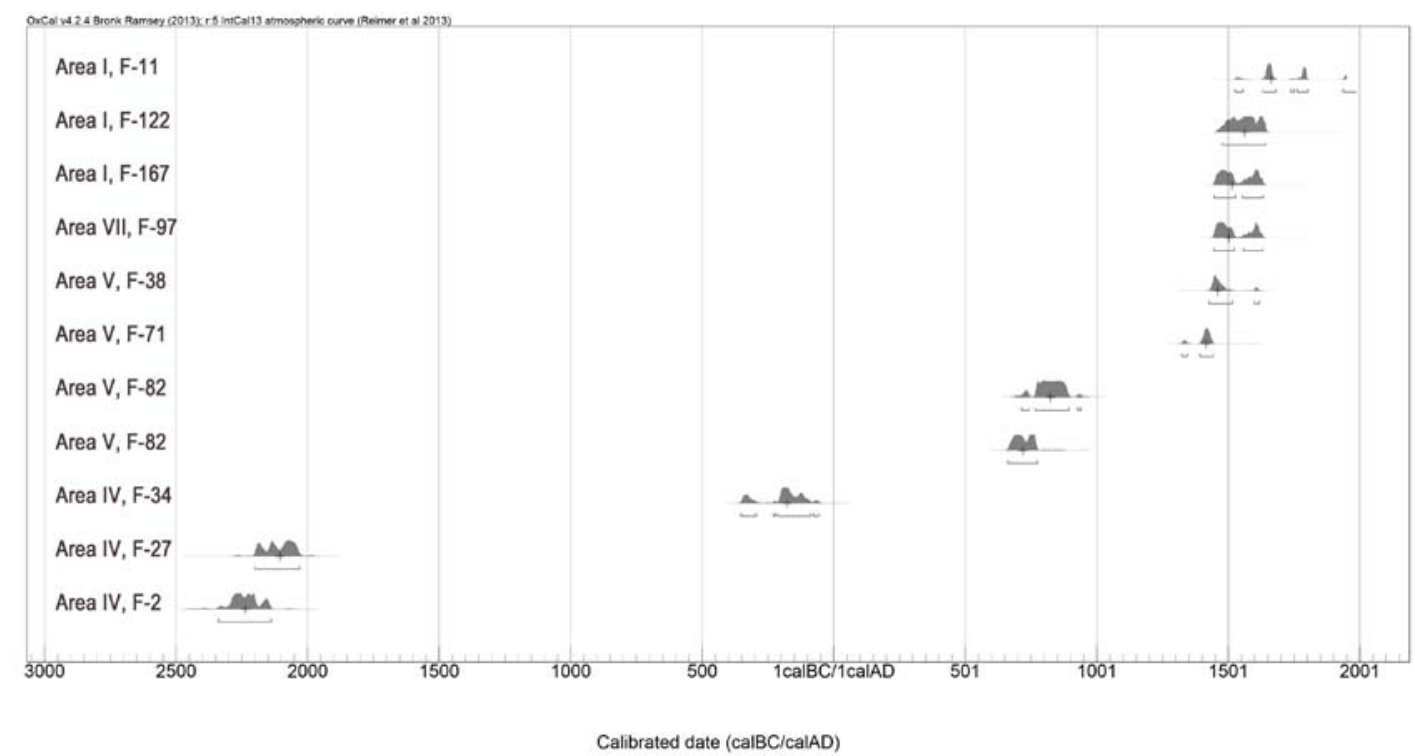

Figure 4. Calibration probability curves (2 sigma) for 11 AMS dates from Dragover (3MN298) features, calibrated using the OxCal 4.2 program and the IntCal13 curve (Bronk Ramsey 2009, 2015; Reimer et al. 2013).

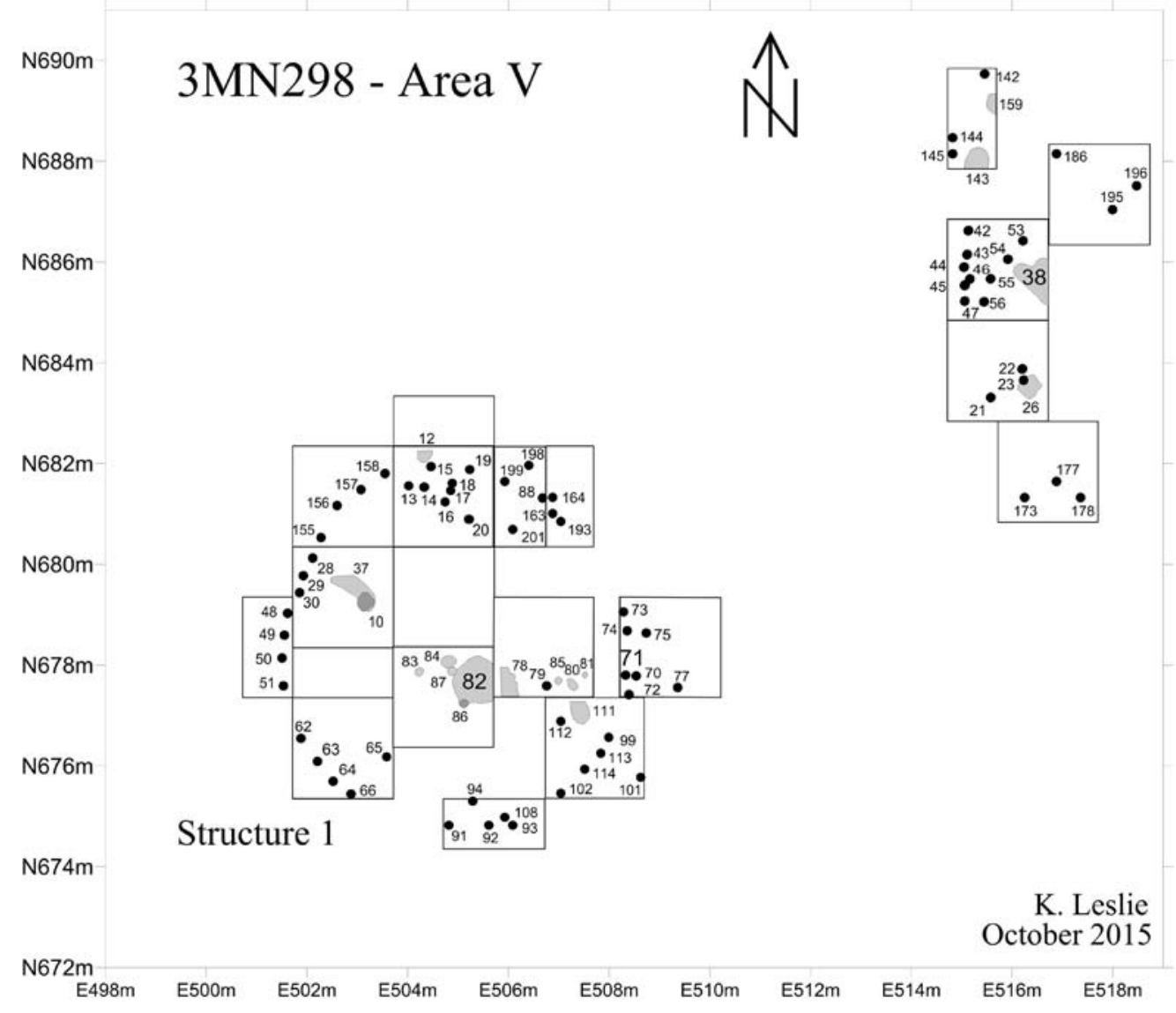

Figure 5. Feature plan for 3MN298 Area V excavations (larger numbers indicate dated features). 

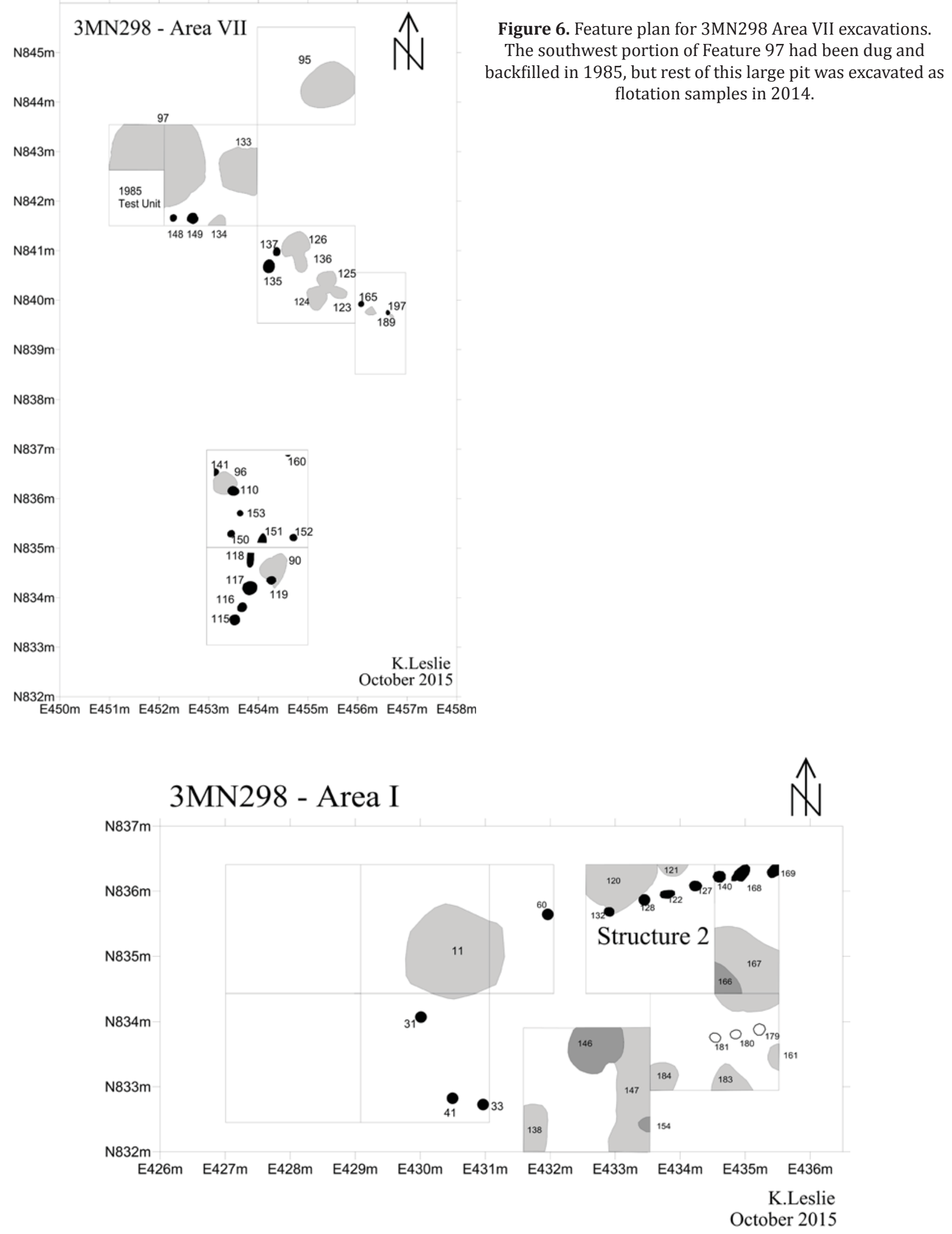

Figure 7. Feature plan for 3MN298 Area I excavations. 


\section{Methods}

Flotation samples from the Dragover site were processed in the field lab during the 2013 and 2014 Society Training Programs or at the Arkansas Archeological Survey's Henderson State University Research Station in a SMAP-type machine (Figure 8). Carbonized material from selected features was removed from the heavy fractions and sent to Macrobotanical Analysis along with the flotation light fractions. Funding is always limited, and a priority list of key features (pits, hearths and structure postholes, and FCR clusters) from 2013 excavation areas was drawn up by Mary Beth Trubitt for botanical analysis and dating. Well-preserved faunal material was found only in a few of the Area VII features excavated in 2014, with pit Feature 97 (Figure 9) the only context yielding a significant amount of preserved bone. A sample of animal bone sorted from heavy fraction flotation samples excavated from the west half of Feature 97 was selected by Mary Beth Trubitt and Katie Leslie, and conveyed to Lucretia Kelly for analysis. Excavation of this portion of the pit was by depth (zones 1-6 and basal), and the analysis included samples from each zone except the uppermost (possibly disturbed) zone. Mussel shell from the same feature is currently undergoing analysis by Robert Scott (Arkansas Archeological Survey) and will be reported in a future publication. A small sample of carbonized botanical material from Feature 97 has been analyzed by Leslie Bush and is included here.

$3 \mathrm{MN} 298$ - Area VII

N 841.5 E 452

Feature 109 and Feature 97

West Wall Profile

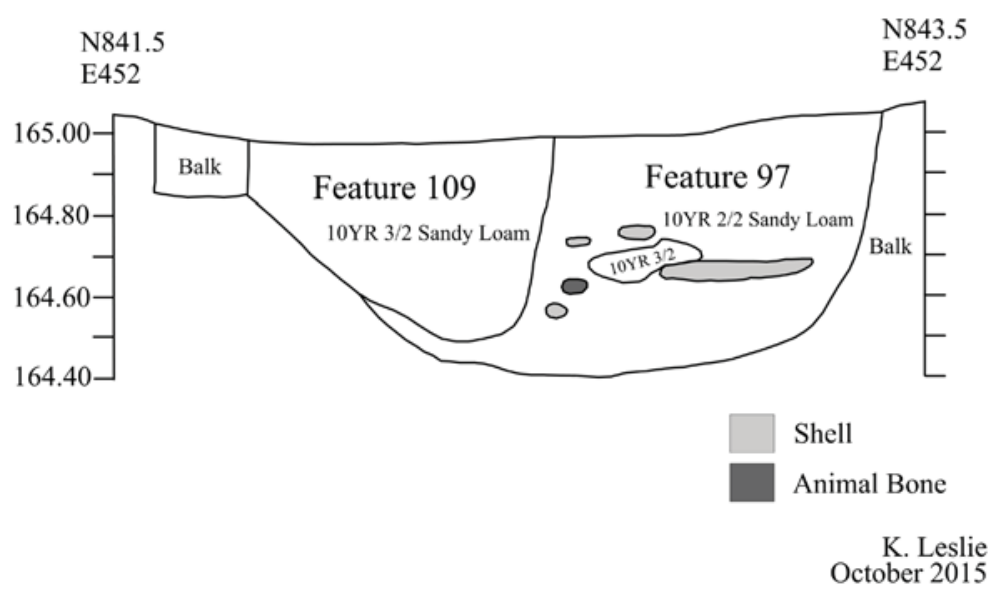

Figure 9. Profile of Feature 109 (representing backfilled test unit) and Feature 97 (refuse pit with well-preserved faunal material).

Twenty-six flotation samples representing 638.5 cubic decimeters ("liters") of fill from 20 features were sorted according to standard procedures at the Macrobotanical Analysis laboratory in Manchaca, Texas (Pearsall 2000). Heavy and light fraction materials were combined prior to size-sorting through a stack of graduated geologic mesh for ease of identification. Materials that did not pass through the No. 10 mesh (2 mm square openings) were completely sorted under a microscope at 7-45 X, and all carbonized and semicarbonized botanical remains were counted, weighed, recorded, and labeled. Materials that fell through 
the $2 \mathrm{~mm}$ mesh ("residue") were examined for carbonized and semi-carbonized botanical remains not previously identified in the larger size fraction. Wood charcoal identification was attempted for 20 specimens selected at random from those larger than $2 \mathrm{~mm}$ from each sample. Botanical materials were identified to the lowest possible taxonomic level by comparison to materials in the Macrobotanical Analysis comparative collection and through the use of standard reference works (e.g., Core et al. 1979; Davis 1993; Hoadley 1990; InsideWood 2004-onwards; Martin and Barkley 1961; Panshin and de Zeeuw 1980; Wheeler 2011). Plant nomenclature follows the PLANTS database (USDA, NRCS 2015).

All animal bone in this study was identified by direct comparison to modern osteological collections in Lucretia Kelly's laboratory or housed at the Illinois State Museum Records and Research Facility in Springfield, Illinois. Identification of the faunal remains was made to the most specific taxonomic level possible, given the completeness and portion of the bone present. Nomenclature follows the Integrated Taxonomic Information System (www.itis.gov). If a bone could be only tentatively identified to a certain taxon, it was given a "cf." designation. As is common in zooarcheology (Driver 1992; Reitz and Wing 2008; Uerpmann 1973), when a mammal or bird bone cannot be identified to a taxon more specific than class, it is placed in a size category where possible. The categories of large, medium, and small are based on the size of the major bones in a skeleton. For each bone identifiable to the level of class or to a more specific taxon, recorded information included element name, side, portion present and completeness, epiphyseal fusion, tooth wear and eruption, and type and placement of modification (weathering, gnaw marks, cut marks, staining, and burning). For the deer bone, locations of cut marks were recorded to aid interpretation of butchery techniques. Epiphyseal fusion and tooth eruption and wear can be used to calculate the relative age of deer (Gilbert 1990; Purdue 1983; Reitz and Wing 2008; Severinghaus 1949).

Following standard zooarcheological quantification practices, both NISP (number of identifiable specimens) and MNI (minimum number of individuals) are used here. For NISP, if two or more fragments could be glued or fitted together, they were counted as one (except teeth are counted separately even if still in the mandible or maxilla, for possible calculation of food utility indices). MNI was figured for each species for the feature as a whole because of potential mixing throughout the feature. MNI was calculated by counting the most frequently-occurring element after dividing lefts from rights. Completeness, portion, age, and, in some cases, size were taken into consideration.

\section{Results: Botanical Remains from Early Components in Areas II, IV, and V}

Fifteen flotation samples (302.4 cu. dm.) from ten features from Areas II, IV, and V were analyzed for macrobotanical remains (Table 2). Seven of these are interpreted as Late Archaic or Woodland period (Fourche Maline) features, including several that have now been AMS dates (two FCR clusters from Area IV with a combined median probability of 2172 B.C., a posthole from Area IV with a median probability of 179 B.C., and a hearth from Area $V$ with a combined median probability of A.D. 743). Three additional postholes from Area IV that may be from either Fourche Maline or Caddo components are also discussed here based on the absence of cultivated plant remains and small seeds. With the exception of the Area $V$ hearth, botanical materials from these features are similar, characterized by sparse wood charcoal and moderate quantities of nutshell. Feature 82 contained a large amount of wood charcoal as well as squash, the earliest cultigen identified from the site, several small seeds from wild plants (sida, spurge), and a fragment of cane. 


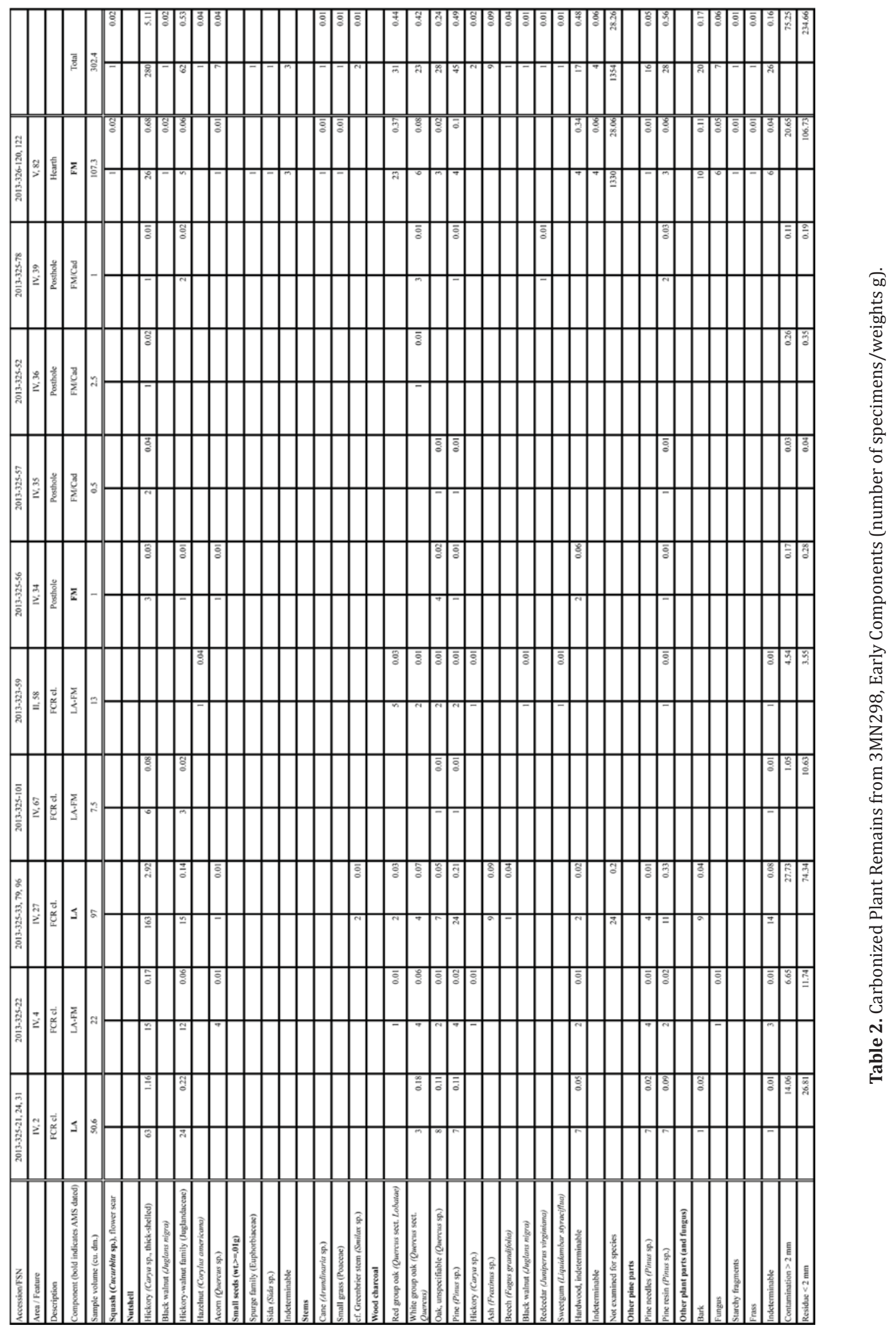




\section{Wood charcoal}

There were 1517 fragments of wood charcoal weighing 30.57 g recovered from early component flotation samples; these are interpreted as fuel wood. Of the 163 fragments for which identification was attempted, 142 could be assigned to genus or species: 82 specimens (58 percent) were oaks, and 45 (32 percent) were pine. Much of the pine came from soil in/around Feature 27, a Late Archaic FCR concentration. Oak predominated in the Feature 82 hearth fill. Little wood charcoal was present in the four postholes (Features 34, 35, 36, and 39), and most had at least two types of wood as well as nutshell. Plant remains in the postholes are interpreted as general site debris rather than primary construction material.

\section{Nut resources}

A total of 351 fragments of nutshell (5.74 g) was recovered from the early features at Dragover. The nutshell is dominated by thick-shelled hickory and Juglandaceae nutshell consistent with hickory (98 percent by weight), but seven fragments of acorn, one of black walnut, and one of hazelnut were also present. Nut uses and nutritional properties are discussed in more detail below. Fats available in the nut resources would have been particularly important to hunter-gatherers who relied on lean meat for a portion of the year (Hall 2000).

\section{Greenbrier vine}

A Late Archaic FCR concentration (F-27) produced two fragments of a monocot stem anatomically consistent with greenbrier, a thorny, thicket-producing vine. Greenbrier has several food uses. Its young shoots are particularly tasty raw or sautéed, and they are also edible after boiling. The bland berries, although not terribly palatable, are at least not toxic to humans (Tull 2013). Historic records of aboriginal greenbrier uses focus on the starchy tubers. Cherokees, Choctaws, and Houma people of Louisiana dried greenbrier tubers and ground them into flour (Moerman 1998). Intriguingly, Melvin Gilmore (1933:126) records greenbrier vines as the main ingredient in a decoction that, when sprinkled on the bed of a couple, would cause them to quarrel and possibly separate. Greenbrier vines were also used in Native basketry in California (Moerman 1998).

\section{Squash}

The only possible domesticated plant identified in early component features at Dragover was squash recovered from Feature 82, a hearth or fire pit with ash deposits excavated in Area V. The squash flower scar, sometimes called a torus (Figure 10 ), has been directly dated and returned a 2-sigma calibrated age range of A.D. 715-940, with a median probability of A.D. 825. Although domestication status cannot be assessed from the flower scar, wild cucurbits are not reported for the Ouachita Mountains, making the squash either a domesticate or an import (Gentry et al. 2013; Smith 1992:82). Domesticated squash and gourd have been found in Late Archaic to Early Woodland period contexts in the Ozarks and in Woodland period components in East Texas (Fritz 1990; Perttula 2008), but domesticated plants have rarely been documented at sites in the Ouachita Mountains before the Caddo period (see Leith 2011 for discussion of Fourche Maline subsistence patterns on the northern edge of the Ouachitas in Oklahoma).

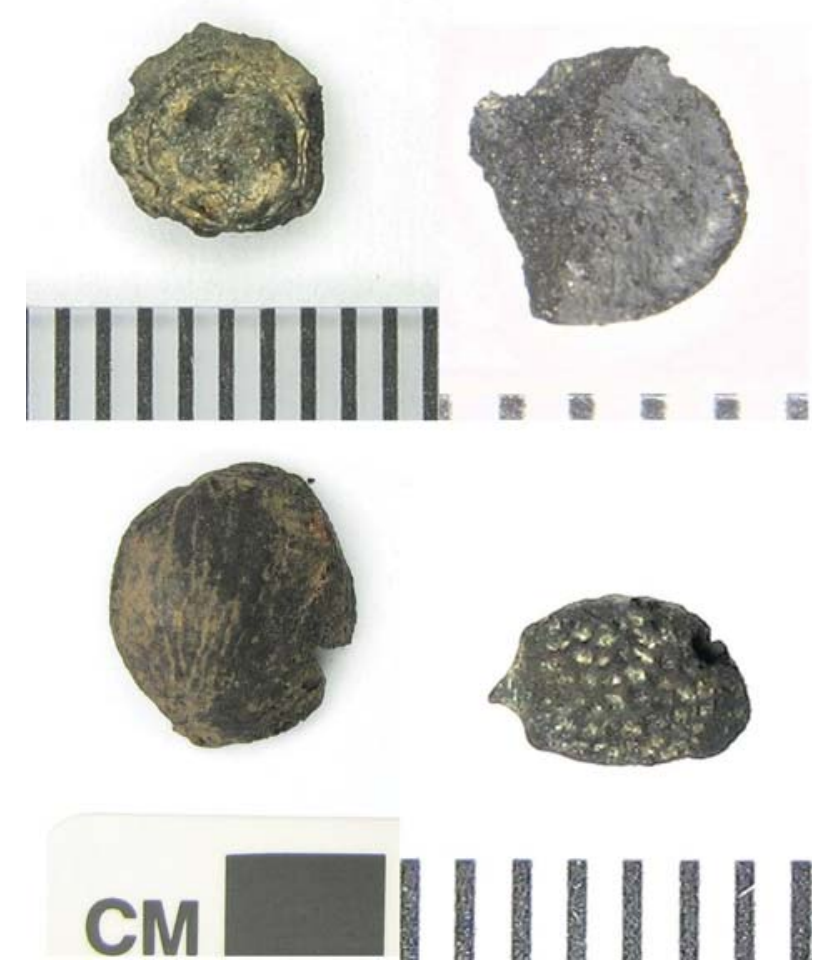

Figure 10. Carbonized plant remains identified from 3MN298 features: top left, squash flower scar from Feature 82 (2013-326-122), scale in mm; top right, common bean fragment from Feature 11 (2013-322-32), scale in mm; bottom left, hickory nutshell from Feature 97 (2014-338-75), scale in cm; bottom right, maypop seed from Feature 11 (2013-322-32), scale in mm (photographs by Leslie Bush). 


\section{Results: Botanical Remains from the Late Component in Areas I, III, V, and VII}

Eleven flotation samples (336.1 cu. dm.) from ten features assigned to the Caddo component in Areas I, III, V, and VII were analyzed for macrobotanical remains (Table 3). Nearly three-quarters of the flotation volume of the Caddo samples comes from Feature 11, a seventeenth century pit from which $235.5 \mathrm{cu}$. dm. of fill were processed and analyzed. Features interpreted as belonging to the Caddo component at Dragover include several different domesticated plants, a variety of small seeds from wild plants, and cane, in addition to nutshell and wood charcoal.

\begin{tabular}{|c|c|c|c|c|c|c|c|c|c|c|c|c|c|c|c|c|c|c|}
\hline AcossionofSN & \multirow{2}{*}{\multicolumn{2}{|c|}{\begin{tabular}{|c|}
$2013-324-111$ \\
10.8
\end{tabular}}} & $2013-326-25$ & \multicolumn{2}{|c|}{$2013.326-65$} & $2013-322.74$ & 2013:-326-100 & \multicolumn{2}{|l|}{$2013-336-101$} & $2013-326-111$ & $2013-32$ & & 2013.322. & $2: 32,60$ & $2014,-3$ & 38.75 & & \\
\hline Ansa/Fetare & & & $v, 10$ & $\frac{v_{1}, 3}{3}$ & & 1,60 & \begin{tabular}{|l|}
$v, 71(5 x, 1)$ \\
\end{tabular} & \begin{tabular}{|l}
$v, 72(\sin : 1)$ \\
\end{tabular} & & $v, 77(15 \times, 1)$ & \begin{tabular}{|l|l|l|l|}
0.8815 \\
\end{tabular} & & 0,11 & & $\frac{\mathrm{VII}, \mathrm{r}}{\mathrm{s}}$ & & & \\
\hline Description & Miste & & FCR \&L. & $\mathrm{P}_{\mathrm{H}}$ & & Posthole & Pesshole & Posahtols & & Posublole & Posth & & Pit & & $\mathrm{Pi}_{\mathrm{i}}$ & & & \\
\hline 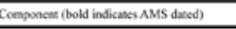 & Cadde & & casto & Cad & & Cudso & Caddo & Costo & & Casdo & Cade & & Cade & & $\mathrm{Cad}$ & & $\overline{T S}$ & al \\
\hline Smple volame (sa.dm) & 305 & & 12 & 38 & & 2 & 3.9 & 0.8 & & 1.5 & 3.95 & & 235.5 & & 8 & & & 6.1 \\
\hline Com (Zes mayx). cepules and ylumes & & & & is & 0.00 & 2001 & & & & & & 0.01 & 55 & 020 & & 0.16 & & 0.52 \\
\hline Com (Zeos angy), kemel & & & 2000 & & 0.05 & & & & & & & 0.05 & 10 & oa & & 0.00 & 33 & 0.41 \\
\hline Squash (Cowartiatasp), riad & & & & & & & 0.04 & & & & & & & & & & & 0.04 \\
\hline 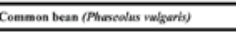 & & & & & & & & & & & - & -5 & 1 & 0.01 & & & & 0.01 \\
\hline Nuthell & & & & & & & & & & & & & & & & & & \\
\hline 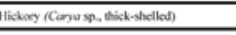 & & 0.01 & 0.0 & 27 & 0.83 & $\begin{array}{ll}10 & 0.11 \\
\end{array}$ & 0.17 & 0.0 .0 & & & E & 0.1 & 2000 & 62.74 & 63 & 2.5 .5 & 3020 & 67.99 \\
\hline 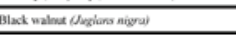 & & & & & 0.06 & & & & & & - & & & & 12 & 0.58 & & 0.64 \\
\hline 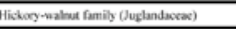 & & & 0.00 & & 0.06 & 0.01 & & & & & E. & 0.01 & 397 & 3.25 & 1 & 0.16 & 415 & 33 \\
\hline 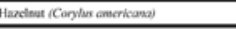 & & & & & & & & & & & - & & & 0.09 & & 0.02 & & 0.09 \\
\hline 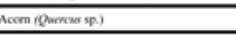 & & & & & & 0.02 & - & & & & - & 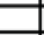 & 14 & 0.05 & & 0.01 & & 0,000 \\
\hline 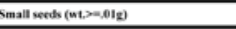 & & & & & & & & & & & & & & & & & & \\
\hline 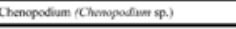 & & & & & & & & & & & & & & & 2 & & & \\
\hline 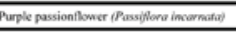 & & & & & & - & - & & & & - & - & 1 & 0.001 & S & 0.2. & & 0.25 \\
\hline 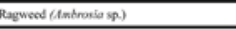 & & & & & & & & & & & & & & & & & & \\
\hline Sindmat (Chumosegyes sp.) & & & & & & & & & & & & & & & & & & \\
\hline 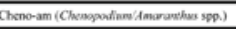 & & & & & & & & & & & 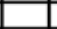 & & & & & & & \\
\hline 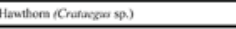 & & & & & & & & & & & & & & 0.00 & & & & 0.02 \\
\hline Legane family (Fabocace) & & & & & & & 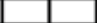 & & & & & & & & & & & \\
\hline 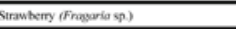 & & & & & & & & & & & & & & & & & & \\
\hline 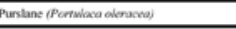 & & & & & & & & & & & & & & & & & & \\
\hline Vemain fierbeso sp) & & & & & & & & & & & & & & & & & & \\
\hline Daisy fanily (Ascrnetesc) & & & & & & & - & & & & & & & & & & & \\
\hline 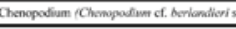 & & & & 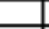 & & & & & & & & & & & & & & \\
\hline Shide-tight (Gatian sp.) & & & & & & & & & & & & & & & & & & \\
\hline 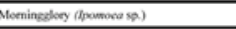 & & & & & & & - & & & & & & & - & & & & \\
\hline Burcloner (MNathazoso sp.) & & & & 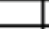 & & 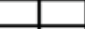 & - & & & & & & & & & & & $\square$ \\
\hline 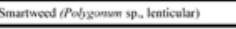 & & & & & & & - & & & & & & & & & & & \\
\hline Grape (minis spe) & & & & & & & & & & & & & & & & & & \\
\hline Indeterminable & & & - & L & - & $\square$ & $\square$ & - & & E & - & - & E & $\square$ & 3 & 0.06 & 1 & 0.00 \\
\hline Stems & & & & & & & & & & & & & & & & 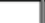 & & \\
\hline Cane (Anundianria s.p.) & & 0.01 & & $\omega_{-1}$ & 0.01 & 0.01 & 0.00 & 2 & & 0.0 .1 & & & & 0.00 & to & 0.33 & की & 0.47 \\
\hline Small grass (Poscex) & & & & & $\square$ & $\square$ & $\square$ & & & E & E & - & - & 0.00 & & $\square$ & t & 0.01 \\
\hline Hebastous & & 0.01 & & - & & - & $-1,2>$ & & & - & - & & & $\square$ & & & & 0.01 \\
\hline Wood chareal & & & & & & & & & & & & & & & & & & \\
\hline 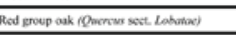 & & 0.04 & 0.00 & 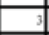 & 0.02 & 0.004 & 0.01 & - & & E & - & 0.04 & & 0.05 & & & 23 & 0.21 \\
\hline 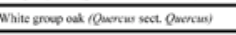 & & & & 10 & 0.25 & 0.05 & 0.02 & 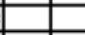 & & - & 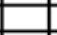 & $\square$ & 10 & 022 & & $0.5 \mathrm{~s}$ & 46 & 1.07 \\
\hline OAk unspecifable (Quercus sp.) & & & & & 0.01 & 0.01 & & & & & & & 11 & 0.22 & & & 13 & 0.24 \\
\hline 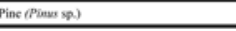 & & & & $\mathrm{E}$ & 0,07 & 0.15 & 0,001 & $\frac{a x}{a}$ & & 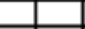 & - & 0.02 & 12 & 0.24 & 12 & 0.58 & 42 & 1.00 \\
\hline Hickoy f(Canuspa) & & & & & & 0.01 & $\square$ & & & & 11 & 0.02 & & 0.02 & & 0.02 & 14 & 0.00 \\
\hline 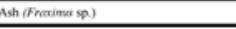 & 12 & 0.25 & & & & & & & & & & 0.01 & & & & & 13 & 0.20 \\
\hline 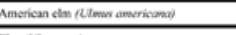 & & 0.07 & & 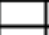 & & & - & & & & - & & E & 0.01 & & & & 0.00 \\
\hline $\lim \left(U_{\ln m}\right.$ sp. $)$ & & & & & & $\pi$ & & & & & - & 0.01 & & 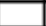 & & & & 0.01 \\
\hline 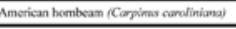 & & & & & & & & & & & & 0.14 & & & & & & 0.14 \\
\hline 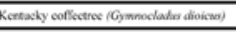 & & & 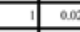 & & & & E & & & & & & & & & & & 0.02 \\
\hline Holly possumbuw (Max sp.) & & & & & & & & & & & E & 0.01 & & - & & & & 0.01 \\
\hline 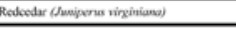 & & & & & & & $0,0.1$ & & & & & & & & & & & 0.01 \\
\hline Hindmood, indsceminatib & & & 0.00 & & & & 0.01 & & & & & & & 0.01 & & & & 0.03 \\
\hline Not ceamined fer species & \begin{tabular}{l|l}
+40 & -12 \\
\end{tabular} & 4.14 & - & 24 & $1 ., 43$ & a.04 & $\square$ & & & 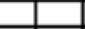 & 20 & 0.22 & 18983 & 2459 & 39:4 & 9.13 & 2838 & \begin{tabular}{|c|c|c|}
3959 \\
\end{tabular} \\
\hline Other pine pars & & & & & & & & & & & & & & & & & & \\
\hline 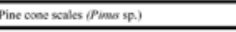 & & & & & & & - & & & & - & 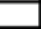 & 2 & 0.01 & & E & & 0.01 \\
\hline Pine nesdles (Pinurs spos) & & & & & & & & & & & & & & 0.01 & & & & 0.01 \\
\hline Pine resin (Pimersp) & & 0.01 & & & 0.07 & & ב & & & & 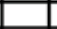 & & 52 & 0.73 & 21 & 0.24 & 73 & 1.12 \\
\hline Other phat parts (and frangus) & & & & & & & - & & & & & & & & & & & \\
\hline Bark & & & & 6 & 1.02 & - & & & & & 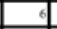 & 0.1 & 41 & 038 & $-3+3$ & 0.05 & 120 & 1.51 \\
\hline Funges & 22 & 0.13 & & & & & & & & & & & & & & 0.02 & 26 & 0.15 \\
\hline Standy fraperents & & & & & & & - & & & & 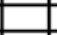 & - & 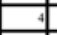 & 0.00 & & - & & 0.00 \\
\hline Tuler & & & & & & & & & & & & & - & & - & 0.58 & & 0.58 \\
\hline Grass hivome (Pouscea) & & & & & & & & & & & & & & 0.01 & & & & 0.01 \\
\hline Rect & & & & & & & & & & & & & 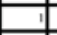 & 0,05 & & & & 0.003 \\
\hline Indetemikable & & & & & 0.03 & -1 & 0.01 & & & & & 0.01 & 111 & 1.9 & 48 & 0.4 & 176 & 24 \\
\hline Cectaninaxios $>2 \mathrm{mmm}$ & & 16.82 & $1.98>$ & & 10.60 & 0.2 & 0.61 & a. & 의 & 0.05 & & 0.2 & $7>$ & 39.4 & & 148509 & & $218.47]$ \\
\hline$R$ Residue $<2 \operatorname{man}$ & & 21.06 & 2.44 & & 8,02 & 0.33 & 0.54 & a & 037 & 0 of & & 0.96 & & 55.15 & & 338.84 & & ans \\
\hline
\end{tabular}

Table 3. Carbonized Plant Remains from 3MN298 Flotation Samples, Late Component (number of specimens/weight g). 


\section{Agricultural products}

Several crop plants were recovered from late component features at the site. Six of the features produced corn, one contained squash rind (F-71), one (F11) yielded a fragmentary common bean, and one (F-88) had a domesticated-type chenopodium seed (goosefoot). Corn consisted of loose kernels and kernel fragments (five features) and cupules and glume fragments (five features). In addition, the fragmentary tip of a corn cob was recovered from the east half of Feature 97 (2014338-21) (Figure 11). Measurements were made of loose corn cupules and the cupules attached to the Feature 97 cob. Loose cupules from Features 11, 38, and 97 had a mean width of $4.3 \mathrm{~mm}$ (range 2.5-7.25, $\mathrm{n}=36$ ) and a mean height or thickness of $1.8 \mathrm{~mm}$ (range 1.1-2.9, $\mathrm{n}=36$ ). The cob fragment had mean cupule widths between 3.9-4.6 $\mathrm{mm}$ and mean cupule heights between 2.6-2.8 for the 6 ranks measured. The cob held 12 rows of kernels and was $11.1 \mathrm{~mm}$ in diameter at the broken end, as measured between the apical notches of lower glumes on opposite sides of the cob (Bird 1994). Corn from 4 features was directly dated using AMS, returning calibrated median probabilities of A.D. 1460, 1503, 1515, and 1663.

Three pieces of squash rind were recovered from posthole Feature 71, associated with Structure 1. These rind fragments are $2.3-2.5 \mathrm{~mm}$ thick, sufficient to meet the "King's Rule" criterion for domestication (Smith 1992:41). They were directly dated using AMS and returned a 2 sigma calibrated age range of A.D. 13241443 , with a median probability of A.D. 1416. A bean fragment was recovered from Feature 11, a seventeenth

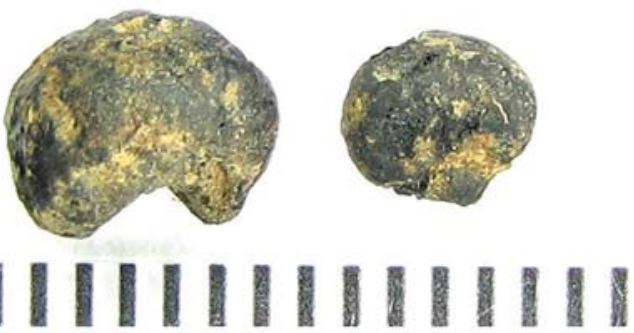
| || ||||||||||||||

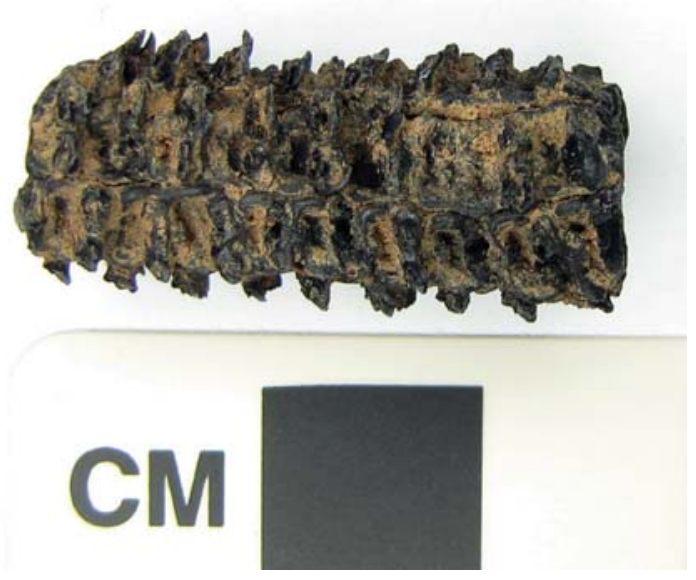

Figure 11. Corn from 3MN298: top, corn kernels from Feature 11 (2013-322-32), scale in mm; bottom, corn cob fragment from Feature 97 (2014-338-21), scale in cm (photographs by Leslie Bush).

century pit (see Figure 10). This age is consistent with the late (post-A.D. 1250) introduction of beans into agricultural systems in the eastern United States (Hart and Scarry 1999).

Cane

Fragments of cane stem were recovered in eight of the ten features assigned to the Caddo component at Dragover (as well as in the Woodland period hearth Feature 82 from Area V). Cane (Arundinaria sp.) is the only North American member of the bamboo tribe, a woody group of the grass family (Diggs et al. 2006:836). A facultative wetlands plant, it grows in moist woods and low areas generally (USDA, NCRS 2015). Although cane is rare today, it was once far more abundant (Jurney 2012). Native people used cane in many items, including mats, baskets, screens to divide rooms, spear shafts, arrow shafts, pipe stems, blow guns, flutes, and blow tubes used in healing (Moerman 1998:104). Henri Joutel mentions use of cane for torches (Foster 1998:235). Historic accounts of the Hasinai Caddo in East Texas by Francisco Hidalgo, Fray Francisco Casañas de Jesús María, and Isidro Felíx de Espinosa all mention baskets or boxes that they describe as made of reed (Hatcher 1927a:56, 1927b:156), probably referring to cane. The larger cane baskets in Caddo country were used to contain crops in storage areas, and loosely woven baskets were useful in sifting and winnowing (Swanton 1996:157).

\section{Nutshell}

A total of 3,472 fragments of nutshell $(72.30 \mathrm{~g})$ was recovered from the late component features analyzed from Dragover. Nutshell consisted primarily of thick-shelled hickories (see Figure 10), but black walnut, hazelnut, and acorn fragments were also found. Nutritionally, hickory nuts are similar to walnuts, and both are more similar to hazel than to acorn. Acorn is intermediate in fat and starches between the other tree nuts and starchy seeds such as corn (USDA, ARS 2014). 
Traditional hickory nut processing methods used by Cherokees, Choctaws, and members of many other tribes involves pounding the nuts into small pieces and then heating them in water, where the oil can be skimmed off, the nutmeat retrieved from suspension, and the shells allowed to sink to the bottom (Fritz et al. 2001; Moerman 1998). Experiments by archeologists show that this process yields a much larger number of calories per labor invested than does cracking and picking (Talalay et al. 1984:353). Other oily nuts cannot be processed in this manner because either their meats sink along with the shells (hazelnut) or the nuts become bitter (walnut).

Other wild plants

Seeds of wild plants identified in late component features include several from edible fleshy or dry fruits: strawberry, maypop (fruit of the purple passionflower, see Figure 10), grape, and hawthorn. Purslane has edible greens. Other plant seeds may be incidental inclusions (sandmat) or medicinal plants (verbena).

The 22 Chenopodium seeds from Feature 97 fall at the large end of the size range for this genus. At 1.6-2.0 mm in diameter, the seeds compare well to the reported dimensions of 1.3-1.9 mm for Chenopodium simplex and 1.7-2.0 mm for C. berlandieri var. bushianum (Clemants and Mosyakin 2003). Both species are native to Arkansas, although it is not clear whether var. bushianum is included in the Arkansas $C$. berlandieri species (Gentry et al. 2013). Testa thickness has not been measured for chenopodium at the site, but the Feature 97 specimens appear subjectively to be the thick, wild type. The single specimen from Feature 88, in contrast, is consistent with the domesticated variety Chenopodium berlandieri spp. jonesianum in having a thin testa and truncate margin morphology; it is $1.3 \mathrm{~mm}$ in maximum diameter. Thin seed coats sometimes occur in wild populations of chenopodium (Smith 1992:148-149), so the domestication status of all chenopodium in the late component at Dragover cannot be determined from a single specimen. Nonetheless, the presence of a domestic type chenopodium indicates an avenue for future research at the site.

\section{Wood charcoal}

A total of 3,003 fragments of wood charcoal ( $42.78 \mathrm{~g}$ ) was recovered in the late component feature samples. Of the 165 fragments for which identification was attempted, 161 could be assigned to genus or species: 82 specimens ( 51 percent) were oaks, and 42 (26 percent) were pine. As in the early component samples, the remaining wood charcoal consisted primarily of hickory and ash. Five of the samples were from postholes (Features 60, 71, 72, 77, and 88), most associated with Structure 1 in Area V. As with the postholes from earlier components, these features appear to be filled with general site debris rather than the remains of construction material.

\section{Results: Animal Bone from Late Component in Area VII}

A total of 1636 vertebrate remains (453.4 g) make up the assemblage analyzed by Lucretia Kelly from the west half of Feature 97, a large pit in Area VII that has an AMS date with a calibrated median probability of A.D. 1503. The analyzed assemblage represents a sample (about 20 percent by weight) of the animal bone recovered from this pit. The results are discussed by animal class and feature zone to see if any differences can be observed as the pit was filled with refuse (Table 4). Remains from four of the five vertebrate classes are present (amphibians are not represented).

Some weathering, carnivore and rodent gnawing, and staining have taken place that indicate bones were exposed to the elements (Table 5). A portion of the assemblage has been burned. In some cases, only singeing is evident and that may reflect a cooking method such as roasting, where only a small part of exposed bone was directly subjected to fire. Bones that are blackened by fire are not usually exposed to high temperatures or are protected by soft tissue when exposed (David 1990). Calcined bone indicates exposure to higher temperatures or more prolonged exposure to fire. More blackened bone than calcined bone was recovered. 


\begin{tabular}{|c|c|c|c|c|c|c|c|c|}
\hline & $338-71$ & $338-72$ & $338-75$ & $338-76$ & $338-78$ & $338-79$ & \multirow{2}{*}{ Total NISP } & \multirow{2}{*}{ Total MNI } \\
\hline & Zone 2 & Zone 3 & Zone 4 & Zone 5 & Zone 6 & Basal & & \\
\hline \multicolumn{9}{|l|}{ Mammals } \\
\hline Didelphis virginiana & & 1 & & & & & 1 & 1 \\
\hline Scalopus aquaticus & & & & 1 & & & 1 & 1 \\
\hline Neotoma floridana & & 1 & 1 & & & & 2 & 1 \\
\hline Sciurus sp. & & 3 & 3 & & & & 6 & 1 \\
\hline cf. Tamias striatus & & 1 & & 3 & & & 4 & 1 \\
\hline Odocoileus virginianus & 7 & 13 & 18 & 5 & 13 & 5 & 61 & 3 \\
\hline large mammal & & & 12 & 12 & 8 & & 32 & - \\
\hline medium-large mammal & 41 & 6 & 3 & 19 & 1 & 10 & 80 & - \\
\hline medium mammal & & 1 & 7 & 5 & & & 13 & - \\
\hline medium-small mammal & & 7 & & 5 & & & 12 & - \\
\hline small mammal & & & & & & & 0 & - \\
\hline Total mammal & 48 & 33 & 44 & 50 & 22 & 15 & 212 & 8 \\
\hline Mammal or Bird & & 1 & & 5 & & & 6 & - \\
\hline \multicolumn{9}{|l|}{ Birds } \\
\hline Meleagris gallopavo & & & & 1 & & & 1 & 1 \\
\hline large bird & & & & 1 & & & 1 & - \\
\hline medium-large bird & & & & & 1 & & 1 & - \\
\hline medium-small bird & & 2 & & & & & 2 & - \\
\hline Total bird & 0 & 2 & 0 & 2 & 1 & 0 & 5 & 1 \\
\hline \multicolumn{9}{|l|}{ Fish } \\
\hline Lepisosteus sp. & 1 & 3 & & 1 & & & 5 & - \\
\hline Lepisosteus osseus & & & & 1 & & & 1 & 1 \\
\hline Catostomidae & & & & 1 & & & 1 & - \\
\hline Moxostoma sp. & & 1 & 1 & 7 & & & 9 & 1 \\
\hline Ictaluridae & & & & 1 & & & 1 & 1 \\
\hline Esox sp. & & & 1 & & & & 1 & 1 \\
\hline Centrarchidae & 1 & 1 & 8 & 6 & & 3 & 19 & - \\
\hline Micropterus sp. & & & & & & 1 & 1 & 1 \\
\hline Lepomis sp. & & & 3 & 4 & & & 7 & 2 \\
\hline Aplodinotus grunniens & 3 & & 5 & 1 & & 2 & 11 & 1 \\
\hline indeterminate fish & 5 & 18 & 75 & 55 & & 13 & 166 & - \\
\hline Total fish & 10 & 23 & 93 & 77 & 0 & 19 & 222 & 8 \\
\hline \multicolumn{9}{|l|}{ Reptiles } \\
\hline Terrapene sp. & 1 & 2 & 1 & 3 & & & 7 & - \\
\hline Terrapene cf. carolina & & & 1 & & 1 & & 2 & 1 \\
\hline Chrysemys complex & & 1 & 1 & & & & 2 & 1 \\
\hline Testudines & 16 & 34 & 14 & 42 & 3 & 3 & 112 & - \\
\hline Serpentes & & & & 2 & & & 2 & - \\
\hline Colubridae & & 3 & 1 & 1 & & 1 & 6 & 1 \\
\hline Total Reptiles & 17 & 40 & 18 & 48 & 4 & 4 & 131 & 3 \\
\hline Indeterminate Vertebrates & 114 & 127 & 298 & 402 & 3 & 116 & 1060 & - \\
\hline Total Vertebrates & 189 & 226 & 453 & 584 & 30 & 154 & 1636 & 20 \\
\hline
\end{tabular}

Table 4. Summary of Fauna from 3MN298 Feature 97 (2014-338). 


\begin{tabular}{|c|c|c|c|c|c|c|c|c|}
\hline & $\mathrm{Z} 2$ & $\mathrm{Z3}$ & $\mathrm{Z} 4$ & $\mathrm{Z5}$ & $\mathrm{Z6}$ & Basal & Total & $\%$ NISP \\
\hline Unburned & 173 & 208 & 393 & 494 & 10 & 127 & 1405 & 85.9 \\
\hline Burned black & 11 & 12 & 55 & 59 & 18 & 21 & 176 & 10.7 \\
\hline Calcined & 5 & 6 & 5 & 31 & 2 & 6 & 55 & 3.4 \\
\hline Weathered & 6 & 5 & 5 & 2 & 3 & 3 & 24 & 1.5 \\
\hline Gnawed & -1 & 2 & 1 & 1 & - & - & 4 & 0.2 \\
\hline Cut Marks & 1 & 1 & -1 & 3 & 1 & 1 & 7 & 0.4 \\
\hline \multicolumn{9}{|c|}{ Taphonomic Effects for Animal Classes: } \\
\hline & Weight (g) & Unburned & Burned Black & Calcined & Weathered & Gnawed & Cut Marks & \\
\hline Mammal & 303.4 & 176 & 31 & 5 & 21 & 3 & 5 & \\
\hline Mammal/Bird & 0.3 & 6 & -1 & -1 & 1 & - & - & \\
\hline Bird & 38.1 & 4 & 1 & - & & 1 & 1 & \\
\hline Fish & 12.4 & 214 & 5 & 3 & 1 & -1 & 1 & \\
\hline Reptile & 47.3 & 121 & 7 & 3 & 1 & - & -1 & \\
\hline $\begin{array}{l}\text { Indeterminate } \\
\text { vertebrate }\end{array}$ & 51.9 & 884 & 132 & 44 & $\mathrm{~N} / \mathrm{A}$ & N/A & $\mathrm{N} / \mathrm{A}$ & \\
\hline Total & 453.4 & 1405 & 176 & 55 & 24 & 4 & 7 & \\
\hline
\end{tabular}

Table 5. Feature 97 Taphonomy. Taphonomic Feature Effects by Feature Zone.

\section{Mammals}

The middle zones $(3,4$, and 5) of pit Feature 97 yielded the most diverse taxa of mammals, with three to five taxa being identified. Deer (Odocoileus virginianus) is the largest and most likely the main economic mammal. The other taxa identified include small-medium sized mammals such as mole (Scalopus aquaticus), opossum (Didelphis virginiana), tree squirrel (Sciurus sp.), wood rat (Neotoma floridana), and possible chipmunk (cf. Tamias striatus). Tree squirrel and opossum could have been included in the diet, but it is likely that mole, wood rat, and possibly chipmunk are intrusions because they are burrowing animals. The other three zones contained only deer.

A total of 61 deer NISP was recovered from the feature, representing 3 MNI (Table 6). The deer specimens are scattered throughout the pit with zone 4 containing the largest number $(n=18)$ and zone 5 and basal zone containing the fewest (five each). When viewing the deer remains as a single unit, a slightly lower percentage of extremity parts are present (56 percent) than what would be expected if an entire deer skeleton was present (67 percent). Axial elements are represented in a slightly higher percentage (32 percent as compared to 28 percent expected) and appendicular elements are present in almost three times the expected percentage (12 percent as compared with 5 percent expected).

A mandible with molars 2 and 3 was recovered from zone 3 . The wear on the teeth was moderate to heavy indicating an individual about 4 1/2-5 1/2 years of age at death (Schwartz and Schwartz 1981; Severinghaus 1949). Five vertebral fragments (cervical) from zone 4 are very porous and are unfused. This individual was very young because vertebral centra fuse around two months of age (Purdue 1983). These fragments are only slightly larger than cervical vertebrae from a comparative skeleton of a full-term fetal deer. Since fawns are born in Arkansas between late March and late June (Sealander 1979:258), this deer was probably taken in the late spring or early summer months. There is an additional individual represented by a thoracic (zone 5) and a cervical vertebra (basal zone) with fused centra, but unfused centra epiphyses are present. These indicate an individual older than two months but younger than 2-3 years of age.

No bone tools are present. One antler tine tip was recovered from zone 3, but it could not be determined if it had been used as a tool such as a lithic flaker. Five deer specimens exhibit cut marks, most likely from butchering activity (Figure 12). Five deer specimens, all from zones 5 and 6, exhibit singeing that could be an indication of roasting meat. Non-human taphonomic processes include weathering ( $\mathrm{n}=15)$ and rodent and carnivore gnawing $(n=2)$. They were distributed throughout the pit rather than being concentrated in one or two zones. 


\begin{tabular}{|c|c|c|c|c|c|c|c|c|}
\hline & Standard & $\mathrm{Z} 2$ & $\mathrm{Z3}$ & $\mathrm{Z4}$ & $\mathrm{Z5}$ & Z6 & Basal & Total \\
\hline \multicolumn{9}{|l|}{ Extemities } \\
\hline Antler & 2 & 5 & 1 & - & - & - & - & 6 \\
\hline Astragalus & 2 & - & - & - & - & - & - & - \\
\hline Atlas & 1 & - & - & - & - & - & - & - \\
\hline Axis & 1 & - & - & - & - & - & - & - \\
\hline Calcaneum & 2 & - & - & - & - & - & - & - \\
\hline Cuboid & 2 & - & - & - & - & - & - & - \\
\hline Greater Cuneiform & 2 & - & - & - & - & - & - & - \\
\hline Hyoid & 2 & - & - & - & - & - & - & - \\
\hline Lateral maleolus & 2 & - & - & - & - & - & - & - \\
\hline Lesser Cuneiform & 2 & - & - & - & - & - & - & - \\
\hline Lunate & 2 & - & - & - & - & - & - & - \\
\hline Magnum & 2 & - & - & - & - & - & - & - \\
\hline Mandible & 2 & - & 1 & - & - & 5 & - & 6 \\
\hline Metacarpal & 2 & - & - & - & - & - & - & - \\
\hline Metacarpal, vestigial & 4 & - & - & - & - & - & - & - \\
\hline Metatarsal & 2 & - & - & 3 & - & 3 & - & 6 \\
\hline Patella & 2 & - & - & - & - & - & - & - \\
\hline Phalanx 1 & 8 & - & - & 1 & 1 & - & 1 & 3 \\
\hline Phalanx 2 & 8 & - & - & 1 & - & - & - & 1 \\
\hline Phalanx 3 & 8 & - & - & - & - & - & - & - \\
\hline Phalanx, vestigial & 24 & - & - & - & - & - & - & - \\
\hline Pisiform & 2 & - & - & - & - & - & - & - \\
\hline Scaphoid & 2 & - & - & - & - & - & - & - \\
\hline Skull & 25 & - & - & 1 & - & - & - & 1 \\
\hline Tooth & 32 & 1 & 2 & 1 & 1 & - & - & 5 \\
\hline Triquetral & 2 & - & - & - & - & - & - & - \\
\hline Unciform & 2 & - & - & - & - & - & - & - \\
\hline Total & 147 & 6 & 4 & 7 & 2 & 8 & 1 & 28 \\
\hline$\%$ & $67.10 \%$ & & & & & & & $56.00 \%$ \\
\hline \multicolumn{9}{|l|}{ Axial } \\
\hline Innominate & 2 & - & - & - & - & - & 1 & 1 \\
\hline Rib & 26 & - & 5 & 4 & - & 1 & 1 & 11 \\
\hline Sacrum & 1 & - & - & - & - & - & - & - \\
\hline Scapula & 2 & 1 & - & - & - & - & - & 1 \\
\hline Sternebra & 7 & - & - & - & - & - & - & - \\
\hline Vertebra, cervical & 5 & - & - & - & - & - & 1 & 1 \\
\hline Vertebra, thoracic & 13 & - & - & - & 2 & - & - & 2 \\
\hline Vertebra, lumbar & 6 & - & - & - & - & - & - & - \\
\hline Total & 62 & 1 & 5 & 4 & 2 & 1 & 3 & 16 \\
\hline$\%$ & $28.30 \%$ & & & & & & & $32.00 \%$ \\
\hline \multicolumn{9}{|l|}{ Appendicular } \\
\hline Humerus & 2 & - & 1 & $=$ & - & - & $=$ & 1 \\
\hline Radius & 2 & - & 1 & - & - & 1 & - & 2 \\
\hline Ulna & 2 & - & - & - & - & - & - & - \\
\hline Femur & 2 & - & - & 1 & - & 1 & - & 2 \\
\hline Tibia & 2 & - & - & - & 1 & - & - & 1 \\
\hline Total & 10 & - & 2 & 1 & 1 & 2 & - & 6 \\
\hline$\%$ & $4.60 \%$ & & & & & & & $12.00 \%$ \\
\hline Total & 219 & 7 & 11 & 12 & 5 & 11 & 4 & 50 \\
\hline \multicolumn{9}{|l|}{ Not used } \\
\hline Metapodial & - & - & 2 & - & - & 2 & 1 & 4 \\
\hline Sesamoid & - & - & - & 1 & - & - & - & 1 \\
\hline Vertebra, unspecified & - & - & - & 5 & - & - & - & 5 \\
\hline Total not used & - & - & 2 & 6 & - & 2 & 1 & 11 \\
\hline Total Deer Remains & & 7 & 13 & 18 & 5 & 13 & 5 & 61 \\
\hline
\end{tabular}

Table 6. Deer Elements from Feature 97, Site 3MN298. 


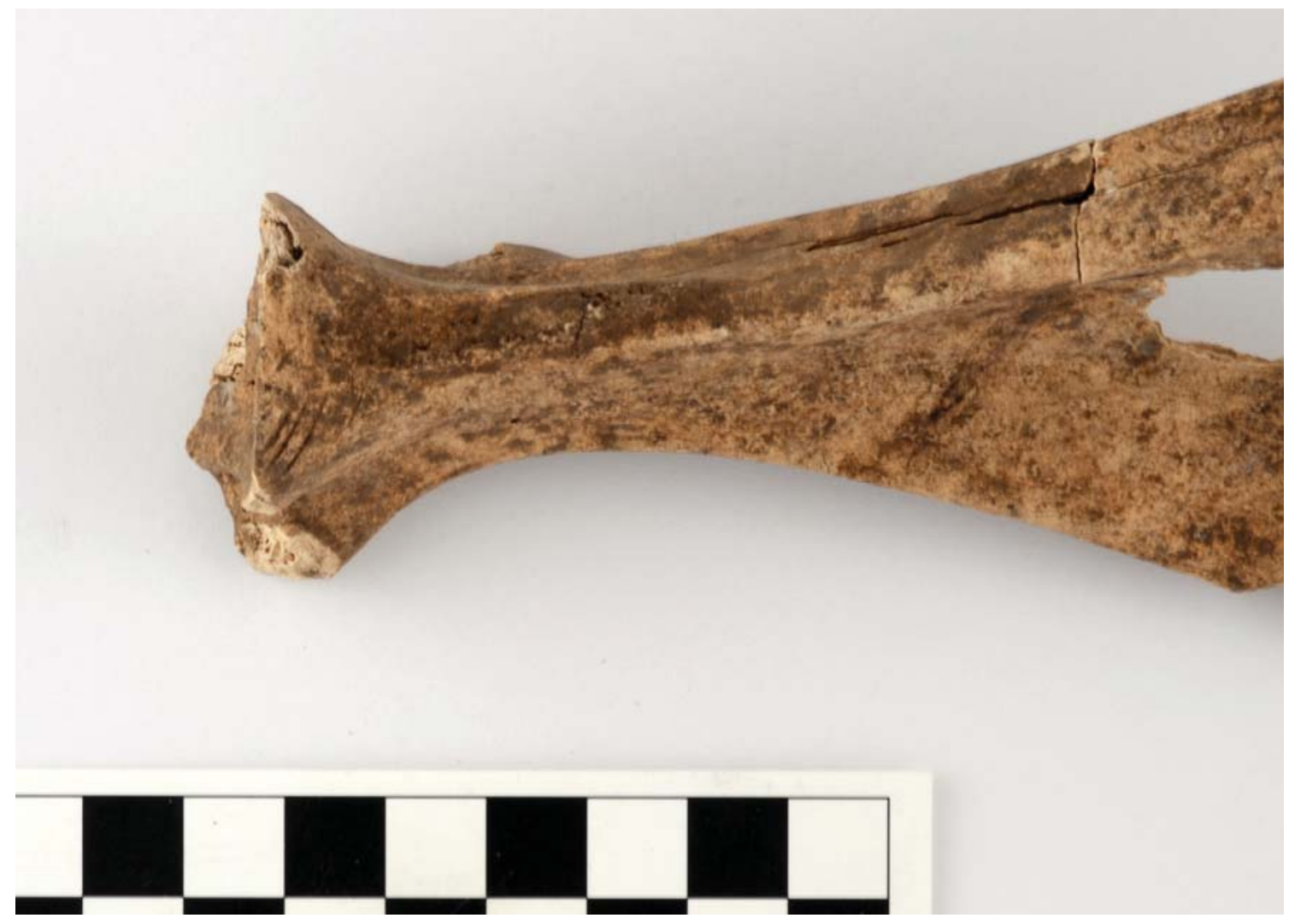

Figure 12. Deer scapula from Feature 97, zone 2 (2014-338-71), with cut marks on glenoid from butchering, scale in cm (photograph by Katie Leslie).

Birds.

Only five bird NISP were recognized and only one taxon identified, turkey (Meleagris gallopavo). The one turkey specimen, a sternum, came from zone 5. It had light cut marks but also carnivore puncture marks.

Fish

Fish remains make up the largest number of NISP from the feature with 222, however 75 percent of the NISP could not be identified below the class level. Ten taxa were identified. These include longnose gar (Lepisosteus osseus), gar (Lepisosteus sp.), redhorse sucker (Moxostoma sp.), sucker family (Catostomidae), catfish family (Ictaluridae), pike (Esox sp.), sunfish family (Centrarchidae), bass (Micropterus sp.), sunfish (Lepomis sp.), and freshwater drum (Aplodinotus grunniens) (Figure 13). Centrarchids make up the largest percentage of the identified fish specimens (48.2 percent NISP) and 50 percent MNI. All but one of the taxa where MNI could be calculated had a MNI of one. Only Lepomis sp. had a MNI of two.

Most of the fish specimens are from small individuals. The longnose gar is of average size and is represented by a parasphenoid that has cut marks presumably made by dismembering the head from the body of the fish. The single bass specimen is weathered. The majority of the fish remains would likely not have been recovered without the use of flotation because the bones are so small.

\section{Reptiles}

Reptile remains, particularly turtle, were fairly numerous throughout the feature. Most of the turtle remains are small fragments of carapace or plastron that could not be identified further. Common box turtle (Terrapene cf. carolina) and box turtle (Terrapene sp.) were recovered from all zones but the lowest two (6 and basal). Two skeletal elements of a turtle in the Chrysemys complex of water turtles (which includes the 
genera of Chrysemys, Pseudemys, Graptemys, and Trachemys; Ernst and Barbour 1989) were recovered from zones 3 and 4. None of the turtle specimens are from large individuals. Snake (Serpentes) remains (seven vertebrae and one rib) were recovered from four zones $(3,4,5$, and basal). Six of the eight vertebrae are from non-venomous snakes (Colubridae).

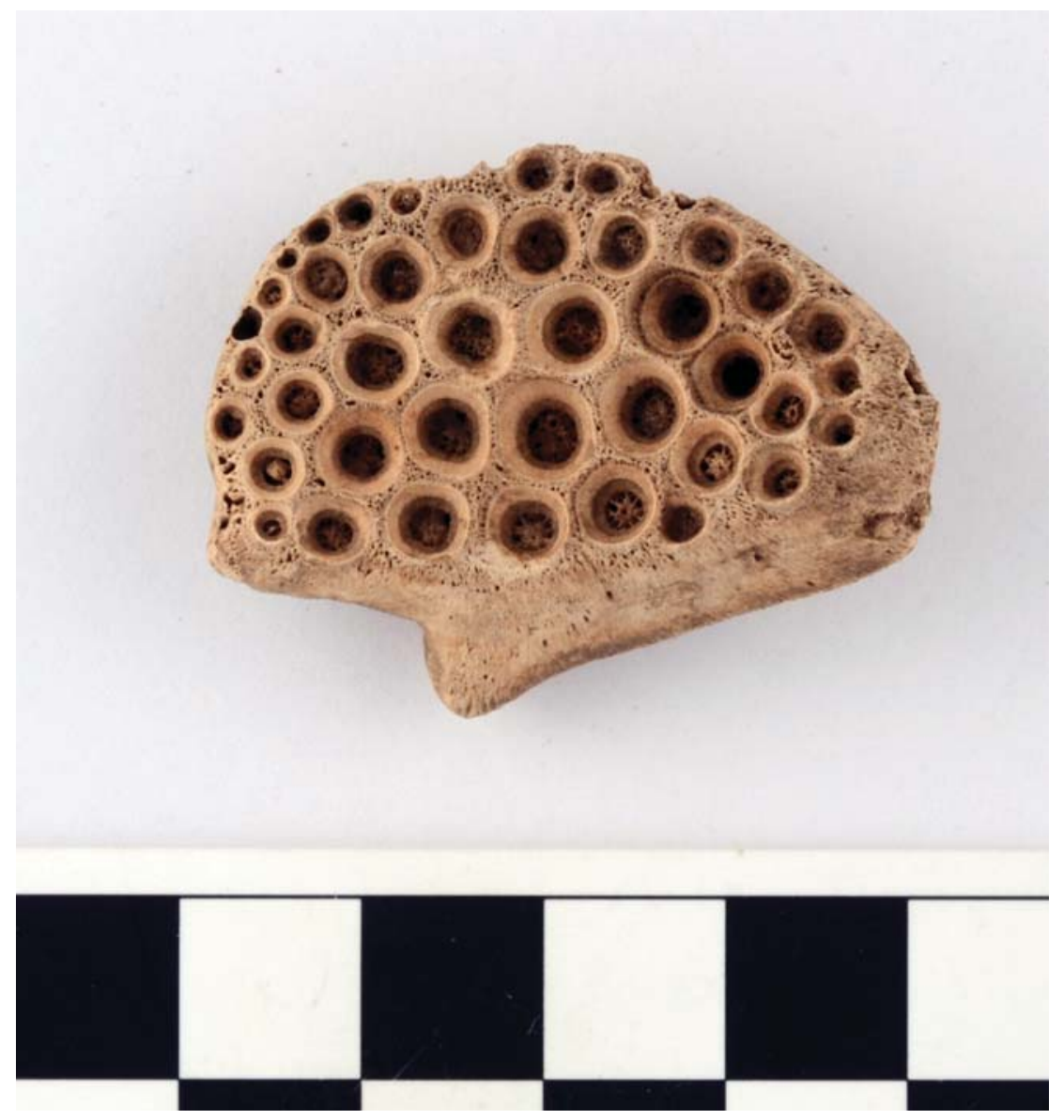

Figure 13. Freshwater drum pharyngeal from Feature 97, zone 5 (2014-338-76), scale in cm (photograph by Katie Leslie).

\section{Discussion: Plant and Animal Foods at Dragover}

Analysis of plant and animal remains from two pit features excavated at Dragover (3MN298) in the 1980s yielded a short list of taxa: plants included hickory and walnut shell, cane, grape seed, persimmon, and a wild seed from the bean family, but no cultigens; and animals included deer, turkey, fox squirrel, box turtle, snapping turtle, bullfrog, longnose gar, bowfin, channel catfish, shiners, chubs, topminnows, water snake, and freshwater mussels (Bennett et al. 1986:60-67). Our more extensive analyses of features excavated in 20132014 from the site has added plant and animal taxa to these lists, most importantly documenting cultivated plants. Squash now dated to the ninth century A.D. is the earliest cultigen present. Corn, squash, a bean, and a domesticated chenopodium seed came from features dating between the fifteenth and seventeenth centuries A.D.

Samples from the late component (Caddo period) contain nearly all the plant taxa of the features from the earlier components. In addition, the later samples produced remains of three crop plants and numerous small seeds. There is also an increase in non-wood plant density between the earlier and later component features (Figure 14). Cooking fires would have been necessary throughout the occupation 
sequence. The increased density of non-wood plants may reflect an actual increase in plant food use, but better preservation in the more recent deposits or the destruction or dispersion of earlier plant material by later occupations may have contributed to the apparent increase.

The taxa composition of the wood and nutshell in the samples from earlier and later component features are generally similar. Wood charcoal recovered to date is interpreted as fuel wood. In this context, the similarly between earlier and later components suggests a continuity in the composition of the local forest composition from Late Archaic through Caddo periods.

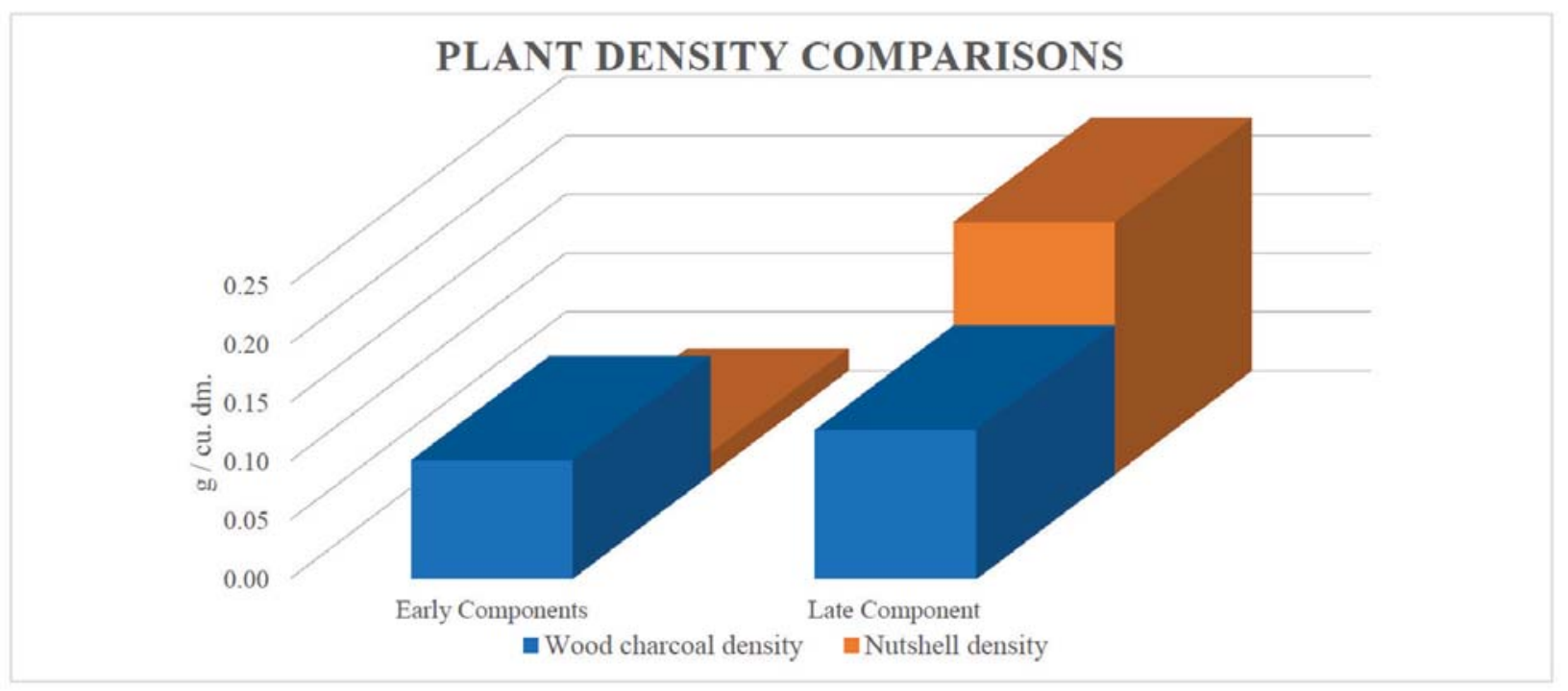

Figure 14. Comparison of plant densities between early and late components at Dragover (wood charcoal and nutshell density per cubic decimeter of processed fill by weight).

The relatively large number of animal remains recovered from Feature 97 is in part attributed to good preservation and to the use of flotation, but this was the only feature excavated with significant amounts of preserved faunal material. While 35 percent of the 1636 vertebrate NISP could be identified to animal class (Figure 15), only 9 percent could be identified to a taxonomic level of family, genus, or species. Mammals were identified most frequently, with deer remains present in the greatest number and at least three individuals present. The other mammals are small-medium in size and represented by only one individual each. Fish are frequent in the assemblage but most are small individuals and would not have made a great contribution to the diet. The same can be said of the turtles. Turkey is the only bird present and is represented by a single specimen.

The ancestral Caddos utilized the environments close to the site including the Ouachita River and the adjacent wooded areas. The young deer in Feature 97 was probably taken in the late spring or early summer months. The fish and turtles would also indicate exploitation in warmer months of the year. Because of their small size it is likely they would have been captured by net or basket. Weathering and animal gnawing on some of the bones indicates they may have lay exposed on the surface for a time before being deposited into the pit as refuse. Some bones were burned, so this may have included cleaned-out fire pits. The types of animals represented are most likely from general domestic activity rather than a specialized activity such as feasting. 


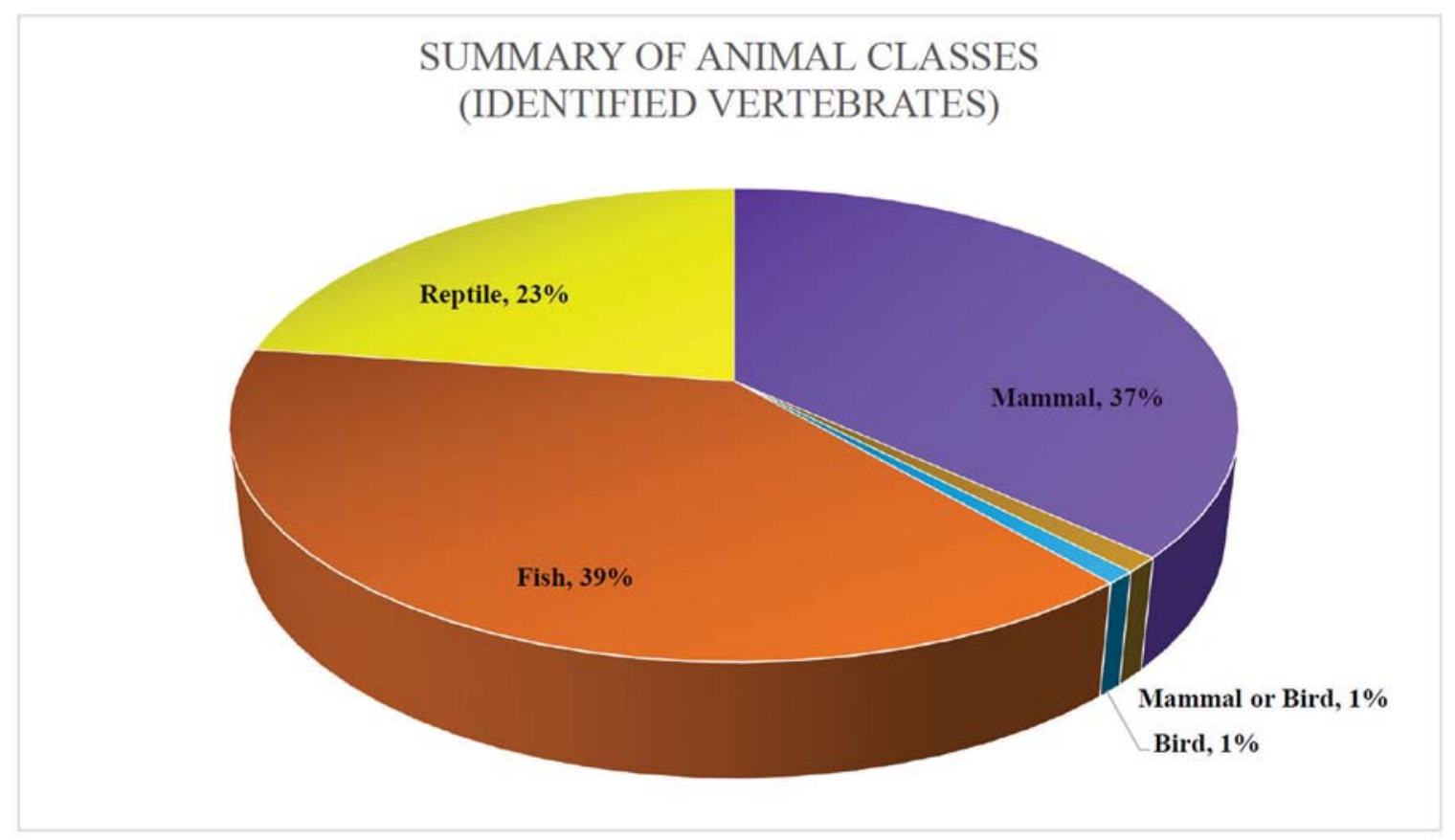

Figure 15. Summary of animal classes identified in Feature 97, 3MN298 (NISP percentages for identified vertebrates).

\section{Regional Comparisons}

The spectrum of plants recovered at the Dragover site is typical for the time periods represented, with wood charcoal and nutshell dominating the early components, and crops - especially corn - and small seeds present in the later samples. Dimensions of the corn cupules recovered to date from the site fall into the small end of the range of archeological Caddo corn (Table 7). It is worth remembering that 53 of the 89 cupules measured at the site come from the tip end of a single cob. Cupules and associated kernels at the tip (distal) end of corn cobs are typically smaller than those of the mid-shaft, and this is one possible explanation for the small mean cupule dimensions. Another possibility is that corn from Dragover was a smaller variety than that grown for consumption at other Caddo sites.

Late prehistoric corn assemblages are compared in Table 8 according to the number of rows of kernels present on cobs at the site. With only one cob of 12 rows available from Dragover, comparison to other assemblages is limited to the observation that 12-rowed corn is typical on Mississippian and Caddo sites. Seven cob fragments from Winding Stair on the upper Little Missouri River in the Ouachita Mountains consisted of three 12-row and four eight-row specimens (Williams 2000). Both cob fragments recovered from the Ramos Creek site in southeastern Oklahoma were from 12-row corn (Dowd and Regnier 2014). Fort Ancient sites more typically have higher percentages of eight-rowed corn. Although different varieties of corn often have different ranges of row numbers, row number varies within corn varieties, and more than one variety can have the same typical range.

Historical accounts offer more detailed information on historical varieties of Caddo corn. One of the earliest accounts of corn in the Caddo area comes from Casañas, writing to the Viceroy of Mexico in 1691:

Among the seeds which the Indians plant in the proper season, is corn of two kinds, which they plant in abundance. One kind matures in a month and a half and the other in three months. (Hatcher 1927c:211) 


\begin{tabular}{|c|c|c|c|c|}
\hline Site & $\begin{array}{r}\text { Cupule mean } \\
\text { width }(\mathrm{mm})\end{array}$ & $\begin{array}{r}\begin{array}{r}\text { Cupule mean } \\
\text { thickness } \\
(\text { height })(\mathrm{mm})\end{array} \\
\end{array}$ & $\begin{array}{r}\text { Number of cupules } \\
\text { measured }\end{array}$ & Reference \\
\hline Dragover (3MN298) loose cupules (Fea. 11, 38, 97) & 4.3 & 1.8 & 36 & this article \\
\hline Dragover (3MN298) Fea. 97 cob fragment & 4.3 & 2.7 & 53 & this article \\
\hline Oak Hill Village (41RK214) Fea. 86* & 4.8 & 3.1 & $\sim 10,220$ & Elson et al. 2004 \\
\hline Musgano (41RK19) & 4.8 & 2.2 & 1380 & Bush 2014a \\
\hline 41PN175 Fea. 87 & 4.8 & 2.3 & 39 & Bush 2015 \\
\hline W. A. Ford (41TT852) & 5 & 2.9 & 10 & Bush 2014b \\
\hline Shelby Mound (41CP71) & 5 & 2.8 & 52 & Bush 2014c \\
\hline Pine Tree Mound (41HS15) & 5.3 & 2.3 & 106 & Bush 2012 \\
\hline Stallings Ranch (41LR297) & 5.4 & 3.1 & 35 & Bush 2008 \\
\hline $41 \mathrm{CP} 183$ & 5.4 & 2.1 & 24 & Sherman et al. 2015 \\
\hline Eli Moores (41BW2) Cob \#1 & 5.8 & 3.5 & 21 & Perttula (ed.) 2014 \\
\hline Henry M. (41NA60) Lot 292 & 6.1 & 1.9 & 20 & Perttula et al. 2010 \\
\hline Ramos Creek (34MC1030), all others & 6.4 & 3.1 & 87 & Dowd and Regnier 2014 \\
\hline Winding Stair (3MN496) & 6.5 & $\mathrm{n} / \mathrm{a}$ & 256 & Williams 2000 \\
\hline Eli Moores (41BW2) loose cupules & 7.1 & 3.4 & 60 & Perttula (ed.) 2014 \\
\hline Indian Creek 2 (41SM404) Fea. 1 & 7.1 & 3.4 & 8 & Nash 2012 \\
\hline Sha'chahdínnih (41MR211)*** & 7.2 & 2.8 & 229 & Goldborer 2002 \\
\hline Ramos Creek (34MC1030) Lot 448.3 & 8.4 & 3.3 & 5 & Dowd and Regnier 2014 \\
\hline \multicolumn{5}{|c|}{$\begin{array}{l}\text { *Mean of measurements given in Table } 91 \text {. Number of cupules estimated from } 108 \text { measurable cobs, row numbers distributed as given in Tabl } \\
91 \text {, assuming } 10 \text { measurable cupules per rank. }\end{array}$} \\
\hline
\end{tabular}

Table 7. Corn cupule (Zea mays) measurements from selected Caddo sites.

\begin{tabular}{|c|c|c|c|c|c|c|c|c|}
\hline \multirow{2}{*}{ Site } & \multicolumn{6}{|c|}{ Row numbers, by percentage of cobs present } & \multirow{2}{*}{ Site Type } & \multirow{2}{*}{ Reference } \\
\hline & 8 & 10 & 12 & 14 & 16 & $\mathrm{~N}$ & & \\
\hline Moundville (1TU500), I & $21 \%$ & $42 \%$ & $13 \%$ & $17 \%$ & $8 \%$ & 24 & Mississippian & Scarry 1986:Table 9.1 \\
\hline Moundville (1TU500), II/III & $29 \%$ & $50 \%$ & $20 \%$ & $1 \%$ & & 119 & Mississippian & Scarry 1986:Table 9.2 \\
\hline Angel (12Vgl) & $35 \%$ & $42 \%$ & $22 \%$ & $1 \%$ & & 367 & Mississippian & Wagner 1991:Table 1 \\
\hline George C. Davis (41CE19) & $36 \%$ & $46 \%$ & $18 \%$ & & & 325 & Caddo & Jones 2000:249 \\
\hline Oak Hill Village (41RK214) & $39 \%$ & $41 \%$ & $17 \%$ & $3 \%$ & & 109 & Caddo & Elson et al. 2004 \\
\hline Pine Tree Mound (41HS15) & $43 \%$ & $29 \%$ & $29 \%$ & & & 7 & Caddo & Bush 2012 \\
\hline Winding Stair (3MN496) & $57 \%$ & & $43 \%$ & & & 7 & Caddo & Williams 2000 \\
\hline Ramos Creek (34MC1030) & & & $100 \%$ & & & 2 & Caddo & Dowd and Regnier 2014 \\
\hline Dragover (3MN298) Fea. 97 & & & $100 \%$ & & & 1 & Caddo & this article \\
\hline Sha'chahdínnih (41MR211) & & $100 \%$ & & & & & Caddo & Goldborer 2002:Appendix Table 2 \\
\hline Roseberry (46MS53a) & $94 \%$ & $5 \%$ & & & & 902 & Fort Ancient & Wagner 1987:Table 6.3 \\
\hline Incinerator (33MY57) & $77 \%$ & $10 \%$ & $7 \%$ & $1 \%$ & & 88 & Fort Ancient & Wagner 1987:Table 6.3 \\
\hline Canter's Caves (33JA3) & $69 \%$ & $17 \%$ & $11 \%$ & $2 \%$ & & 84 & Fort Ancient & Wagner 1987:Table 6.3 \\
\hline Kettle Hill Cave (33FA2) & $78 \%$ & $18 \%$ & $4 \%$ & & & 77 & Fort Ancient & Wagner 1987:Table 6.3 \\
\hline
\end{tabular}

Table 8. Corn Cob assemblages at selected Late Prehistoric Agricultural Sites in the Eastern US. 
Antoine-Simon Le Page du Pratz lived among Native people in Louisiana and Mississippi in the early eighteenth century, learning the Natchez language. Although not Caddoan speakers, the Natchez are Mississippian descendants who probably had similar corn traditions. Le Page du Pratz (1774:Book III, Chapter I) describes six types of corn that ripened at different times and had kernels of varying colors and sizes.

Squash was the only pre-maize crop recovered from Dragover, and it is among the earliest domesticates in the Americas (Kistler et al. 2015). The single domestic-type specimen of chenopodium was recovered from Feature 88 in a Caddo context. As discussed above, the chenopodium specimens from Feature 97 appear to be thick-testaed, the wild type of C. simplex or C. berlandieri var. bushianum. Similarly, the Polygonum specimen is lenticular in shape and clearly not the trigonous Polygonum erectum that was domesticated in some areas.

Cultivation of native starchy seeds of chenopodium, maygrass, little barley, and knotweed contemporary with Fourche Maline occupations is known elsewhere in the Central Mississippi and Ohio River valleys, roughly in the areas of the later Mississippian and Fort Ancient cultures (Johannessen 1993:Figure 5-1). Starchy seed cultivation was either absent or less important in other areas of the Eastern Woodlands. In the Lower Mississippi River valley, there is little evidence of native starchy seed use until Marksville times, but even then cultivation is "not on the scale of their trading partners to the north" (Fritz 2000:238). Only in the northern part of the Lower Mississippi River valley does pre-corn cultivation seem to have been important, as at the Taylor Mounds site in southeastern Arkansas (Fritz 2000:238). In contrast to the situation in the Lower Mississippi River valley, intensive cultivation of native seed crops is apparent in the Arkansas River valley (Fritz 2000:238). Although the investigators could not be sure the seeds were cultivated, all four types of native starchy seeds were recovered from the Hardman site, which lies farther down the Ouachita River in the Coastal Plain (Fritz 1993). In the Ouachita Mountains, the Winding Stair site in the upper Little Missouri River valley produced chenopodium and possible knotweed. Only chenopodium is present at Amos, in the upper Caddo River valley (Williams 2000). The dearth of these starchy seeds at Dragover is consistent with previous findings in the Ouachita Mountains.

Few Caddo sites from the Ouachita Mountains region have faunal remains preserved and reported (Dellinger and Dickinson 1941; Early 1988:140-142), so a regional comparison is not yet possible. A few Caddo sites along the Ouachita River to the south can be compared as well as a Woodland site in the Ouachita Mountains to the north. The Wild Violet site, located in Logan County on a tributary of the Petit Jean River, had a large but highly fragmented faunal assemblage recovered from the Woodland occupation (Kelly 2015). While there may be some bias in what taxa were identified because of taphonomic issues, the inhabitants of the Wild Violet site focused much of their exploitation of fauna from terrestrial habitats and on deer in particular. There was some utilization of the adjacent aquatic habitats as evinced by a few fish vertebra, aquatic turtles (soft-shell and Chrysemys), beaver, and freshwater mussel. In general, the few upland Woodland sites in Arkansas that have had faunal analyses conducted (Kelly 2011a; Styles et al. 1985; see also Jackson and Scott 2002) appear to be very similar in composition. Most of the remains are recovered from midden contexts, and are impacted by taphonomic processes of weathering, animal gnawing, burning, and fragmentation. Terrestrial habitats were exploited most frequently with deer and box turtles the primary taxa taken. They have a variety of small to medium-sized mammals present, but in relatively low numbers. Turkey was the primary bird exploited. Aquatic habitats were exploited to a lesser extent with the presence of a few aquatic turtles and mammals, fish, and freshwater mussels. It is possible the more fragile fish remains have been removed from the assemblages through taphonomic agents.

Turning to more contemporary Caddo sites in the Ouachita River drainage, only a few have had faunal materials preserved and analyzed. The Caddo inhabitants at the Jones Mill site, located to the east of Dragover, exploited nearby edge and aquatic habitats, the latter most likely being the Ouachita River (Trubitt et al. 2011). The assemblage recovered was very similar to Dragover where deer provided the greatest amount of meat, but fish, turtles, and possibly mussels would have also been important sources of animal protein. Birds do not appear to have been exploited to a great extent with turkey the only species identified. In the Gulf Coastal Plain south of the Ouachita Mountains, the Hardman site near the middle Ouachita River had faunal material recovered from Mid-Ouachita to Deceiper phase occupations (ca. A.D. 1450-1700; Styles and White 
1993). The site inhabitants focused their faunal exploitation on deer and fish supplemented by freshwater mussels, aquatic turtles, and a variety of small and medium-sized mammals and terrestrial birds. There was an increase in aquatic taxa between the Mid-Ouachita and Deceiper phase occupations at Hardman. More taxa are found at Hardman than at Dragover, but the taxa are similar in composition between the two sites.

At the Hughes site, further south and east in the Gulf Coastal Plain near the Saline River in central Arkansas, deer and tree squirrels were the most utilized mammals (Kelly 2011b). Birds and fish remains were not prevalent in any of the units while turtle remains made up a slightly larger proportion. Turkey and passenger pigeon were the most utilized birds, and box turtles the most utilized turtle. Three-ridge and Wabash pigtoe were the most prevalent mussels.

Weinand and colleagues (1997) compared fauna from early and late Caddo sites. Their comparison (see Weinand et al. 1997:Table 17) suggests that if a site is located along a larger creek or bayou, more of the vertebrate remains come from aquatic animals or those that inhabit bottomlands (other than deer), but if a site is further from a larger stream, more remains come from deer. They conclude that location with proximity to water plays a greater role in what animals are exploited than whether a site dates to the early or late Caddo period. The fauna from the Hughes site, however, does not fit this hypothesis. The Hughes site is in a bottomland setting of the Saline River. One explanation for this difference is that the Hughes faunal assemblage comes from near the base of a mound and may represent food refuse from mound activity such as feasting or from higher status individuals (Kelly 2011b). Faunal remains from more contexts at Hughes are needed to confirm whether this small assemblage represents specialized activity. Fauna from domestic contexts at Hughes would also help determine if the exploitation pattern of the occupants was different from other Caddo sites located in bottomlands of larger streams and rivers.

The few Caddo sites with analyzed faunal assemblages in the middle to upper Ouachita drainage overall have similar assemblages. Deer is the primary mammal utilized with some small to medium-sized mammals also taken. Birds as an animal class are not well represented but turkey is usually present. If the site is near the river, fish, turtles, and freshwater mussels are well utilized, however, the amount of meat they would have provided would not have been great. The amount of aquatic species in the assemblages appears to increase from the Woodland period. However, it is possible that this may be more a function of taphonomy rather than utilization.

\section{Conclusions}

Analyses of plant and animal remains from excavations at the Dragover site (3MN298) provide new information on the foodways of the people who inhabited the Ouachita Mountains in the past. The dominant food represented in the earlier Archaic and Woodland (Fourche Maline) components is nuts, primarily thick-shelled hickory, but greenbrier was also present, as well as squash, the earliest cultigen recovered at the site. The lack of preserved bone or shell in the early components means there is a lack of evidence on Archaic and Woodland period animal use. Changes are documented in the use of plants for food through time, especially as ancestral Caddo Indians incorporated domesticated crops - corn, squash, beans, and chenopodium or goosefoot - into their diet. Wild plants continue in importance, with several kinds of nuts, wild fruits (strawberry, maypop, grape, hawthorn, and persimmon), and wild greens and grains (purslane, chenopodium) present. In the late component, the people exploited both terrestrial and aquatic resources for food animals, using deer, squirrel, opossum, turkey, several kinds of fish, turtle, bullfrog, snake (perhaps eaten), and freshwater mussel. Many of these foods are still important to contemporary Caddos (Attocknie 2015).

Further analysis will include comparisons of different excavation areas and clarification of community patterns at Dragover and their changes through time. The differential preservation of animal bone across the site is an issue for continued investigation, and the early presence of cultivated squash as well as both wild and domesticated chenopodium in the Caddo component can be pursued further. There are additional samples that can be analyzed once additional funding is secured. Nevertheless, these preliminary results show the research potential of this site and add much needed detail to our picture of Caddo and preCaddo lifeways in the Ouachita Mountains region. 


\section{Acknowledgments}

Thank you to the U.S. Forest Service and to the Arkansas Archeological Society for funding the AMS dates and the archeobotanical and zooarcheological analyses reported here. Collections and records from this project are curated by the Arkansas Archeological Survey at the HSU Research Station in Arkadelphia, Arkansas.

\section{References Cited}

Attocknie, Dana

2015 Caddo Food Traditions Go Beyond Corn, Beans and Squash. Native Oklahoma May, 2015, pp. 11-12.

Bennett, William J., Jr., Anne Frances Gettys, and Aubra Lee

1986 Archeological Testing and Evaluation of 3MN298, Oden Ranger District. Report No. 26. Archeological Assessments, Inc., Nashville, Arkansas.

Bird, Robert McK.

1994 Manual for the Measurement of Maize Cobs. In Corn and Culture in the Prehistoric New World, edited by Sissel Johannessen and Christine A. Hastorf, pp. 5-22. Westview Press, Boulder, Colorado.

Bronk Ramsey, Christopher

2009 Bayesian Analysis of Radiocarbon Dates. Radiocarbon 51(1):337-360.

2015 OxCal Version 4.2, Oxford Radiocarbon Accelerator Unit, University of Oxford. Electronic document, http://c14.arch.ox.ac.uk/embed.php?File=oxcal.html, accessed November 6, 2015.

Bush, Leslie L.

2008 Macrobotanical Remains from the 2005 and 2006 Texas Archeological Society Field Schools at the Gene and Ruth Ann Stallings Site (41LR297). Report submitted to the Texas Archeological Society, by Macrobotanical Analysis, Manchaca, Texas.

2012 Macrobotanical Remains. In Archeology of the Nadaco Caddo: The View from the Pine Tree Mound Site (41HS15), Harrison County, Texas, by Ross C. Fields and Eloise F. Gadus, pp. 728-772. Reports of Investigations No. 164. Prewitt and Associates, Inc., Austin, Texas.

2014a Analysis of the Paleobotanical Remains from the Musgano Site. In The Caddo Archaeology of the Musgano Site (41RK19) in the Sabine River Basin of East Texas, by Timothy K. Perttula, with contributions by Leslie L. Bush, LeeAnna Schniebs, and Robert Z. Selden, Jr., pp. 35-41. Special Publication No. 28. Friends of Northeast Texas Archaeology, Pittsburg and Austin, Texas.

2014b Macrobotanical Plant Remains (Appendix F). In Testing and Data Recovery Excavations at Native American Sites along the U.S. Highway 271 Mount Pleasant Relief Route, Titus County, Texas, by Ross C. Fields, Virginia L. Hatfield, Damon Burden, Eloise Frances Gadus, Michael C. Wilder, and Karl W. Kibler, pp. 613-643. Reports of Investigations No. 168. Prewitt and Associates, Inc. Austin, Texas.

2014c Plant Remains from Shelby Mound (41CP71), Camp County, Texas. Journal of Northeast Texas Archaeology 46:45-55.

2015 Plant Remains from Site 41PN175, Panola County, Texas. In Data Recovery Investigations: Murvaul Creek Site (41PN175), Panola County, Texas, by Arlo McKee, Charles D. Frederick, Timothy K. Perttula, Robert A. Selden, Leslie Bush, Leonard Kemp, Brittney Gregory, Chad Yost, Linda Scott Cummings, Jeffrey R. Ferguson, Michael D. Glascock, Steve Tomka, Leslie Cecil, Caroline Masiello, Xiaodong Gao, Christopher Goodmaster, Virgil Beasley, and Duane E. Peter, pp. 269-286. Report No. 165. Texas Department of Transportation, Environmental Affairs Division, Archeological Studies Program, Austin.

Clemants, Steven E., and Sergei L. Mosyakin

2003 Chenopodium. In Flora of North America North of Mexico, Volume 4, pp. 261-268. New York and Oxford. Electronic document, http://www.efloras.org, accessed February 23, 2015. 
Core, H. A., W. A. Cote, and A. C. Day

1979 Wood Structure and Identification. 2nd edition. Syracuse University Press, Syracuse, New York.

David, B.

1990 How Was This Bone Burnt? In Problem Solving in Taphonomy: Archaeology and Palaeontological Studies from Europe, Africa, and Oceania, edited by S. Solomon, I. Davidson, and D. Watson, pp. 6579. Tempus, Archaeology and Material Culture Studies in Anthropology, Vol. 2. University of Queensland, Australia.

Davis, Linda W.

1993 Weed Seeds of the Great Plains: A Handbook for Identification. University Press of Kansas, Lawrence.

Diggs Jr., George M., Barney L. Lipscomb, Monique D. Reed, and Robert J. O’Kennon

2006 Illustrated Flora of East Texas, Volume One: Introduction, Pteridophytes, Gymnosperms, and Monocotyledons Sida, Botanical Miscellany, No. 26. Botanical Research Institute of Texas, Fort Worth.

Dellinger, Samuel C., and Samuel D. Dickinson

1941 Excavation of the Adair Site, Garland County, Arkansas. WPA Project No. 665-63-3-170. Draft manuscript on file, Arkansas Archeological Survey, Fayetteville.

Dowd, Elsbeth, and Amanda Regnier

2014 Archaeological Investigations at the Ramos Creek Site (34MC1030), McCurtain County, Oklahoma. Final Report submitted to U.S. Forest Service, Ouachita National Forest. Report on file at the Oklahoma Archeological Survey, Norman.

Drennan, Robert D.

1996 Statistics for Archaeologists: A Commonsense Approach. Plenum Press, New York.

Driver, Jonathan C.

1992 Identification, Classification and Zooarchaeology. Circaea 9(1):35-47.

Early, Ann M.

1988 Standridge: Caddoan Settlement in a Mountain Environment. Research Series No. 29. Arkansas Archeological Survey, Fayetteville.

Elson, Katherine M., Christopher Smith, and Timothy K. Perttula

2004 Additional Maize Studies. In The Oak Hill Village Site (41RK214), Rusk County, Texas, by Robert Rogers and Timothy K. Perttula, pp. 323-335. Document No. 030083. PBS\&J, Austin, Texas.

Ernst, C. H., and R. W. Barbour

1989 Turtles of the World. Smithsonian Institution Press, Washington, D. C.

Etchieson, Meeks, and Mary Beth Trubitt

2013 Plans for the $50^{\text {th }}$ Arkansas Archeological Society Training Program. Field Notes, Newsletter of the Arkansas Archeological Society 371:3-8.

Foster, William C. (editor)

1998 The La Salle Expedition to Texas: The Journal of Henri Joutel, 1684-1687. Texas State Historical Association, Austin. 
Foti, Thomas, and C. Theo Witsell

2013 Effects of Physical Factors on the Distribution of Native Flora and Vegetation in the Natural Divisions of Arkansas. In Atlas of the Vascular Plants of Arkansas, edited by Johnnie L. Gentry, George P. Johnson, Brent T. Baker, C. Theo Witsell, and Jennifer D. Ogle, pp. 17-40. PMC Solutions, University of Arkansas, Fayetteville.

Fritz, Gayle J.

1990 Multiple Pathways to Farming in Precontact Eastern North America. Journal of World Prehistory 4(4):387-435.

1993 Archeobotanical Analysis. In Caddoan Saltmakers in the Ouachita Valley: The Hardman Site, edited by Ann M. Early, pp. 159-168. Research Series No. 43. Arkansas Archeological Survey, Fayetteville.

2000 Native Farming Systems and Ecosystems in the Mississippi River Valley. In Imperfect Balance: Landscape Transformations in the Pre-Columbian Americas, edited by David L. Lentz, pp. 225-249. Columbia University Press, New York.

Fritz, Gayle J., Virginia Drywater Whitekiller, and James W. McIntosh

2001 Ethnobotany of Ku-Nu-Che: Cherokee Hickory Nut Soup. Journal of Ethnobiology 21(2):1-27.

Gentry, Johnnie L., George P. Johnson, Brent T. Baker, C. Theo Witsell, and Jennifer D. Ogle

2013 Atlas of the Vascular Plants of Arkansas. PMC Solutions, University of Arkansas, Fayetteville.

Gilbert, B. Miles

1990 Mammalian Osteology. Missouri Archaeological Society, Columbia.

Gilmore, Melvin R.

1933 Some Chippewa Uses of Plants. Papers of the Michigan Academy of Science, Arts and Letters 17:119143.

Goldborer, S. Eileen

2002 Macrobotanical Evidence of Subsistence at Timber Hill. In Finding Sha'chahdinnih (Timber Hill): The Last Village of the Kadohadacho in the Caddo Homeland, edited by Mark L. Parsons, James E. Bruseth, Jacques Bagur, S. Eileen Goldborer, and Claude McCrocklin, pp. 81-86. Archeological Reports Series No. 3. Texas Historical Commission, Austin.

Hall, Grant

2000 Pecan Food Potential in Prehistoric North America. Economic Botany 54(1):103-112.

Hanvey, Vanessa N.

2014 Predictive Modeling of a Caddo Structure in the Ouachita Mountains, Montgomery County, Arkansas. Caddo Archeology Journal 24:43-52.

Hart, John P., and C. Margaret Scarry

1999 The Age of Common Beans (Phaseolus vulgaris) in the Northeastern United States. American Antiquity 64(4):653-658.

Hatcher, Mattie Austin

1927a Descriptions of the Tejas or Asinai Indians, 1691-1722, Part III (Hildago). Southwestern Historical Quarterly 31:50-62.

1927b Descriptions of the Tejas or Asinai Indians, 1691-1722, Part IV (Espinosa). Southwestern Historical Quarterly 31:150-180.

1927c Descriptions of the Tejas or Asinai Indians, 1691-1722, Part I (Casanas de Jesus Maria). Southwestern Historical Quarterly 30:206-218. 
Hoadley, R. Bruce

1990 Identifying Wood: Accurate Results with Simple Tools. The Taunton Press, Newtown, Connecticut.

InsideWood

2004-onwards The InsideWood Database. Electronic document, http://insidewood.lib.ncsu.edu/search, accessed February 4, 2015.

Jackson, H. Edwin, and Susan L. Scott

2002 Woodland Faunal Exploitation in the Midsouth. In The Woodland Southeast, edited by David G. Anderson and Robert C. Mainfort, pp. 461-482. University of Alabama Press, Tuscaloosa.

Johannessen, Sissel

1993 Farmers of the Late Woodland. In Foraging and Farming in the Eastern Woodlands, edited by C. Margaret Scarry, pp. 57-77. University Press of Florida, Gainesville.

Jones, Volney

2000 Appendix. Maize from the Davis Site: Its Nature and Interpretation. In The George C. Davis Site, Cherokee County, Texas, edited by Perry H. Newell and Alex D. Krieger, pp. 241-249. Second edition. Society for American Archaeology, Washington, D.C.

Jurney, David H.

2012 Anthropology of Fire in the Ozark Highland Region. In Proceedings of the 4th Fire in Eastern Oak Forests Conference, edited by Daniel C. Day, Michael C. Stambaugh, Stacy L. Clark, and Callie J. Schweitzer, pp. 12-33. Gen. Tech. Rep. NRS-P-102. United States Department of Agriculture, Forest Service, Northern Research Station. Newtown Square, Pennsylvania. Electronic document, http://www.nrs.fs.fed.us/pubs/41830, accessed February 6, 2015.

Kelly, Lucretia S.

2011a Report of Faunal Remains from Site 3L0769. Report submitted to Tim Mulvihill, University of Arkansas-Fort Smith Research Station, Arkansas Archeological Survey, Fort Smith.

2011b Report of Faunal Remains from the Hughes Mound Site (3SA11). Report submitted to Mary Beth Trubitt, Henderson State University Research Station, Arkansas Archeological Survey, Arkadelphia.

2015 Report of Faunal Remains Recovered from the Wild Violet Site (3L0226). Report submitted to Larry Porter, Winthrop Rockefeller Institute Research Station, Arkansas Archeological Survey, Morrilton.

Kistler, Logan, Lee A. Newsom, Timothy M. Ryan, Andrew C. Clarke, Bruce D. Smith, and George H. Perry

2015 Gourds and Squashes (Cucurbita spp.) Adapted to Megafaunal Extinction and Ecological Anachronism through Domestication. PNAS, published ahead of print November 16, 2015, doi:10.1073/ pnas.1516109112.

Leith, Luther J.

2011 A Re-conceptualization of the Fourche-Maline Culture: The Woodland Period as a Transition in Eastern Oklahoma. Ph.D. dissertation, Department of Anthropology, University of Oklahoma, Norman.

Le Page du Pratz, Antoine-Simon

1774 History of Louisiana or of the Western Parts of Virginia and Carolina: Containing a Description of the Countries that lie on both Sides of the River Mississippi: With an Account of the Settlements, Inhabitants, Soil, Climate and Products. Edited and published by J. S. W. Harmanson with an introduction by Stanley Clisby Arthur. Electronic document, http://www.gutenberg.org/ebooks/9153, accessed February 5, 2015.

Martin, Alexander C., and William D. Barkley

1961 Seed Identification Manual. University of California Press, Berkeley. 
Moerman, Daniel E.

1998 Native American Ethnobotany. Timber Press, Portland.

Nash, Michael A., Timothy K. Perttula, and Linda W. Ellis

2012 National Register of Historic Places Eligibility Testing of Site 41SM404 Within TxDOT's Tyler District, Smith County, Texas. Document No. 110055. Atkins North America, Inc., Austin.

Ouachita Chapter, Arkansas Archeological Society

2014 What's for Supper? Native American Foods in the Ouachita Mountains. Written by John Chapman, Florence Davis, Sue Dawson, Janice Fisher, Mary Ann Goodman, Vanessa Hanvey, Jim Hudgins, Judy Thye, Mary Beth Trubitt, and Ron Zweifel, illustrated by Florence Davis, designed by Tyler Stumpf Ouachita Chapter of the Arkansas Archeological Society, privately printed.

Panshin, A. J., and Carol de Zeeuw

1980 Textbook of Wood Technology: Structure, Identification, Properties, and Uses of the Commercial Woods of the United States and Canada. 4th edition. McGraw-Hill, New York.

Pearsall, Deborah M.

2000 Paleoethnobotany: A Handbook of Procedures. 2nd edition. Academic Press, San Diego, California.

Perttula, Timothy K.

2008 Caddo Agriculture on the Western Frontier of the Eastern Woodlands. Plains Anthropologist 53(205):69-105.

2009 The Caddo Ceramics from the Dragover Site (3MN298) on the Ouachita River on Ouachita National Forest Lands in Montgomery County, Arkansas. The Arkansas Archeologist, Bulletin of the Arkansas Archeological Society 48:1-14.

Perttula, Timothy K. (editor)

2014 The Eli Moores Site, a 17th to Early 18th Century Caddo Site on the Red River, Bowie County, Texas. Special Publication No. 31. Friends of Northeast Texas Archaeology, Austin and Pittsburg, Texas.

Perttula, Timothy K., Leslie L. Bush, LeeAnna Schniebs, Tom Middlebrook, and P. Shawn Marceaux

2010 An Early Historic Caddo Farmstead at the Henry M. Site (41NA60) in Nacogdoches County, Texas. Stephen F. Austin State University Press, Nacogdoches, Texas.

Purdue, James A.

1983 Epiphyseal Closure in White-tailed Deer. Journal of Wildlife Management 47(4):1207-1213.

Reimer, Paula J., Edouard Bard, Alex Bayliss, J. Warren Beck, Paul G. Blackwell, Christopher Bronk Ramsey, Caitlin E. Buck, Hai Cheng, R. Lawrence Edwards, Michael Friedrich, Pieter M. Grootes, Thomas P. Guilderson, Haflidi Haflidason, Irka Hajdas, Christine Hatte, Timothy J. Heaton, Dirk L. Hoffmann, Alan G. Hogg, Konrad A. Hughen, K. Felix Kaiser, Bernd Kromer, Sturt W. Manning, Mu Niu, Ron W. Reimer, David A. Richards, E. Marian Scott, John R. Southon, Richard A. Staff, Christian S. M. Turney, and Johannes van der Plicht

2013 IntCal13 and Marine13 Radiocarbon Age Calibration Curves 0-50,000 Years cal BP. Radiocarbon 55(4):1869-1887.

Reitz, Elizabeth J., and Elizabeth S. Wing

2008 Zooarchaeology. Cambridge University Press, Cambridge, United Kingdom.

Samuelsen, John R.

2014 AMS and Radiocarbon Dating of the Crenshaw Site (3MI6). The Arkansas Archeologist, Bulletin of the Arkansas Archeological Society 52:17-35. 
Scarry, C. Margaret

1986 Changes in Plant Procurement and Production during the Emergence of the Moundville Chiefdom. Ph.D. dissertation, Department of Anthropology, University of Michigan, Ann Arbor.

Schwartz, Charles W., and Elizabeth R. Schwartz

1981 The Wild Mammals of Missouri. University of Missouri Press and Missouri Department of $\backslash$ Conservation, Columbia.

Sealander, J. A.

1979 A Guide to Arkansas Mammals. River Road Press, Conway, Arkansas.

Selden, Robert Z., Jr., and Timothy K. Perttula

2013 Radiocarbon Trends and the East Texas Caddo Tradition (ca. A.D. 800-1680). Southeastern Archaeology 32(1):85-96.

Severinghaus, C. W.

1949 Tooth Development and Wear as Criteria of Age in White-tailed Deer. Journal of Wildlife Management 13:195-216.

Sherman, David L., Leslie Bush, Linda W. Ellis, Tim Griffith, Candace Wallace, and Michael Nash

2015 National Register Testing at 41CP183, a Small Middle Caddo Settlement, Camp County, Texas. Report prepared for Luminant Mining Co., Dallas, Texas, by Blanton \& Associates, Inc., Austin, Texas.

Smith, Bruce D.

1992 Rivers of Change: Essays on Early Agriculture in Eastern North America. Smithsonian Institution Press, Washington, D.C.

Styles, Bonnie W., and Karli White

1993 Faunal Analysis. In Caddoan Saltmakers in the Ouachita Valley: The Hardman Site, edited by Ann M. Early, pp. 145-158. Research Series No. 43. Arkansas Archeological Survey, Fayetteville.

Styles, Bonnie W., James R. Purdue, and Mona L. Colburn

1985 Analysis of Faunal Remains. In The Alexander Site, edited by Thomas E. Hemmings and John H. House, pp. 58-74. Research Series No. 24. Arkansas Archeological Survey, Fayetteville.

Swanton, John R.

1996 Source Material on the History and Ethnology of the Caddo Indians. University of Oklahoma Press, Norman. Originally published 1942 as Bureau of American Ethnology Bulletin 132, Smithsonian Institution, Washington, D.C.

Talalay, Laurie, Donald R. Keller, and Patrick J. Munson

1984 Hickory Nuts, Walnuts, Butternuts, and Hazelnuts: Observations and Experiments Relevant to Their Aboriginal Exploitation in Eastern North America. In Experiments and Observations on Aboriginal Wild Plant Food Utilization in Eastern North America, edited by Patrick J. Munson, pp. 338-359. Prehistory Research Series Volume VI, Number 2. Indiana Historical Society, Indianapolis.

Trubitt, Mary Beth, and Meeks Etchieson

2014 Plans for the 2014 Arkansas Archeological Society Training Program in the Ouachita National Forest. Field Notes, Newsletter of the Arkansas Archeological Society 377:3-5.

Trubitt, Mary Beth, and Katie Leslie

2015 Identity \& Community in the Ouachita Mountains. Electronic document, http://archeology.uark.edu/learn-discover/current-research/ouachita-mountains/, accessed November 11, 2015.

2016 2013-2014 Society Training Program Excavations: The Dates. Field Notes, Newsletter of the Arkansas Archeological Society 389, forthcoming. 
Trubitt, Mary Beth, Meeks Etchieson, and Leslie L. Bush

2014 First Results from 3MN298: The Caddo Community. Field Notes, Newsletter of the Arkansas Archeological Society 377:10-14.

Trubitt, Mary Beth, Kathryn Parker, and Lucretia Kelly

2011 Reconstructing Ancient Foodways at the Jones Mill Site (3HS28), Hot Spring County, Arkansas. Caddo Archeology Journal 21:43-70.

Tull, Delena

2013 Edible and Useful Wild Plants of the Southwest: Texas, New Mexico, and Arizona. Revised edition. University of Texas Press, Austin.

Uerpmann, Hans-Peter

1973 Animal Bone Finds and Economic Archaeology: A Critical Study of 'Osteo-archaeological' Method. World Archaeology 4(3):307-322.

U. S. Department of Agriculture, Agricultural Research Service (USDA, ARS)

2014 USDA National Nutrient Database for Standard Reference. Release 27. Electronic document, http://www.ars.usda.gov/ba/bhnrc/ndl, accessed February 6, 2015.

U. S. Department of Agriculture, Natural Resources Conservation Service (USDA, NRCS)

2015 The PLANTS Database. U. S. Department of Agriculture, Natural Resources Conservation Service, National Plant Data Team, Greensboro, North Carolina. Electronic document, http://plants.usda.gov, accessed February 3, 2015.

Wagner, Gail Elaine

1987 Uses of Plants by the Fort Ancient Indians. Ph.D. dissertation, Department of Anthropology, Washington University, St. Louis, Missouri.

1991 Corn from the Angel Site, Indiana. Paper presented at the 14th Ethnobiology Conference, St. Louis, Missouri.

Ward, G. K., and S. R. Wilson

1978 Procedures for Comparing and Combining Radiocarbon Age Determinations: A Critique. Archaeometry 20(1):19-31.

Weinand, Daniel C., Elizabeth J. Reitz, David B. Kelley, and Melissa Braud

1997 Vertebrate Fauna and Freshwater Mussels. In Two Caddoan Farmsteads in the Red River Valley: The Archaeology of the McLelland and Joe Clark Sites, edited by David B. Kelley, pp. 97-108. Research Series No. 51. Arkansas Archaeological Survey, Fayetteville.

Wheeler, Elizabeth A.

2011 InsideWood - A Web Resource for Hardwood Anatomy. IAWA Journal 32(2):199-211.

Williams, Michele L.

2000 Ethnobotanical Analysis (Winding Stair and Bug Spot). In Forest Farmsteads: A Millennium of Human Occupation at Winding Stair in the Ouachita Mountains, edited by Ann M. Early, pp. 111-122. Research Series 57. Arkansas Archeological Survey, Fayetteville.

Woods, Alan J., Thomas L. Foti, Shannen S. Chapman, James M. Omernik, James A. Wise, Elizabeth O. Murray, William L. Prior, Joe B. Pagan, Jr., Jeffrey A. Comstock, and Michael Radford

2004 Ecoregions of Arkansas (color poster with map, descriptive text, summary tables, and photographs). U.S. Geological Survey, Reston, Virginia. Electronic document, http://cmase.pbworks.com/f/EPA-Ecoregions\%20of\%20Arkansas.pdf, accessed 10 November 2015 